\title{
Entanglement Wedge Reconstruction via Universal Recovery Channels
}

\author{
Jordan Cotler, ${ }^{1}$ Patrick Hayden, ${ }^{1}$ Geoffrey Penington, ${ }^{1}$ Grant Salton, ${ }^{2,1}$ Brian Swingle, ${ }^{1,3,4}$ and Michael Walter ${ }^{5,1}$ \\ ${ }^{1}$ Stanford Institute for Theoretical Physics, Stanford University, Stanford California 94305, USA \\ ${ }^{2}$ Institute for Quantum Information and Matter, Caltech, Pasadena, California 91125, USA \\ ${ }^{3}$ Department of Physics, Harvard University, Cambridge, Massachusetts 02138, USA \\ ${ }^{4}$ Martin Fisher School of Physics, Brandeis University, Waltham, Massachusetts 02453, USA \\ ${ }^{5}$ Korteweg-de Vries Institute for Mathematics, Institute for Theoretical Physics, Institute for Logic, \\ Language and Computation \& QuSoft, University of Amsterdam, 1090 GE Amsterdam, Netherlands
}

(Received 15 October 2018; revised manuscript received 11 February 2019; published 24 July 2019)

In the context of quantum theories of spacetime, one overarching question is how quantum information in the bulk spacetime is encoded holographically in boundary degrees of freedom. It is particularly interesting to understand the correspondence between bulk subregions and boundary subregions in order to address the emergence of locality in the bulk quantum spacetime. For the AdS/CFT correspondence, it is known that this bulk information is encoded redundantly on the boundary in the form of an error-correcting code. Having access only to a subregion of the boundary is as if part of the holographic code has been damaged by noise and rendered inaccessible. In quantum-information science, the problem of recovering information from a damaged code is addressed by the theory of universal recovery channels. We apply and extend this theory to address the problem of relating bulk and boundary subregions in AdS/CFT, focusing on a conjecture known as entanglement wedge reconstruction. Existing work relies on the exact equivalence between bulk and boundary relative entropies, but these are only approximately equal in bulk effective field theory, and in similar situations it is known that predictions from exact entropic equalities can be qualitatively incorrect. We show that the framework of universal recovery channels provides a robust demonstration of the entanglement wedge reconstruction conjecture as well as new physical insights. Most notably, we find that a bulk operator acting in a given boundary region's entanglement wedge can be expressed as the response of the boundary region's modular Hamiltonian to a perturbation of the bulk state in the direction of the bulk operator. This formula can be interpreted as a noncommutative version of Bayes's rule that attempts to undo the noise induced by restricting to only a portion of the boundary. To reach these conclusions, we extend the theory of universal recovery channels to finite-dimensional operator algebras and demonstrate that recovery channels approximately preserve the multiplicative structure of the operator algebra.

DOI: 10.1103/PhysRevX.9.031011

Subject Areas: Gravitation, Quantum Information, String Theory

\section{INTRODUCTION}

The AdS/CFT correspondence is a duality or equivalence between a gravitational theory in $(d+1)$-dimensional asymptotically AdS spacetime and a conformal field theory with one less spatial dimension [1-5]. Roughly speaking, the CFT lives on the boundary of the bulk AdS spacetime, and the quantum state of the boundary CFT is dual to the state of the quantum-gravity theory in the bulk. In the limit of a large number $N$ of degrees of freedom (d.o.f.) and strong coupling in the CFT, the gravity side of the

Published by the American Physical Society under the terms of the Creative Commons Attribution 4.0 International license. Further distribution of this work must maintain attribution to the author(s) and the published article's title, journal citation, and DOI. correspondence can be approximately described by classical Einstein gravity. Hence, AdS/CFT provides a theoretical laboratory to understand how classical spacetime emerges from microscopic d.o.f. and how quantum effects alter the physics, at least in a toy model of quantum gravity. Only certain CFT states correspond to classical geometries in the bulk, and this important class of states occupies much of our attention. For such states, there is an emergent spatial direction in the bulk theory, and understanding how locality in the bulk geometry arises in the boundary theory is a longstanding problem. One way to approach the problem is to ask which regions of the bulk are completely described by a given region of the boundary.

The question above has been phrased in various forms over the years [6,7], and a particularly natural approach is to think operationally. Physical observables are described by Hermitian operators, so the goal becomes 
the identification of all local bulk operators that can be expressed in terms of boundary operators with support only on the boundary subregion. Such operators correspond to those observables in the bulk subregion whose physics can be completely reproduced using boundary observables in a boundary subregion. Significant progress has been made on this problem, starting with the so-called HKLL prescription [2], so named in honor of its authors Hamilton, Kabat, Lifschytz, and Lowe. The method works in certain cases but falls short in general; there are bulk operators that should be expressible on a particular boundary subregion that are inaccessible to the HKLL technique. An example is the operator corresponding to the area of the minimal surface in the bulk that calculates the entropy of the boundary subregion, the so-called Ryu-Takayanagi surface $[8,9]$. For a single hemispherical region in empty AdS, HKLL and related techniques suffice to reconstruct bulk operators in terms of a boundary spherical region, but almost any more-complex scenario falls outside of the purview of HKLL.

The problem of finding the dual to a boundary subregion is at the heart of the subject of bulk reconstruction: Given an operator in the bulk, can one find a representation of this operator acting on a subregion of the boundary? Recently, it has been proposed that for a given subregion $A$ of the conformal boundary, any low-energy bulk operator acting on a spacetime region called the entanglement wedge of $A$ can be reconstructed using only information in $A[6,10,11]$. Roughly speaking, the entanglement wedge of a boundary subregion is the bulk spacetime region between the boundary subregion and its corresponding bulk minimal surface, the aforementioned Ryu-Takayanagi (RT) surface. More precisely, for time-symmetric situations, the entanglement wedge is the set of bulk spacetime points that, due to the bulk light-cone restriction, can be influenced only by the bulk spatial region between the RT surface and the boundary subregion (see below for a fully general definition). The entanglement wedge reconstruction conjecture was strengthened in Ref. [12] and established in tensor network toy models of holography [12-14]. Very recently, it was proved in Refs. $[15,16]$ under the condition that the bulk and boundary relative entropies are exactly equal.

At large but finite $N$, within the framework of bulk effective field theory, one expects only approximate equality of the bulk and boundary relative entropies. When similar situations were studied in the quantum-information theory literature, it was found that algebraic consequences of exact entropic equalities often do not provide qualitatively correct predictions in the approximate case. An important and, in fact, closely related example is the exact saturation of strong subadditivity of the von Neumann entropy, which is known to imply that the underlying state is a quantum Markov chain [17]. Such states have an orderly pattern of correlation in which different subsystems are independent of each other given an appropriate buffer between them. The associated algebraic structure slightly generalizes operator algebra quantum-error correction and is precisely the structure relevant in AdS/CFT [15]. Near saturation of strong subadditivity, however, fails to imply that the state is nearly a quantum Markov chain state for systems of large Hilbert space dimension [18,19]. As we discuss above, a large number of local d.o.f. is essential in AdS/CFT, so the failure of the approximate version of the implication in large Hilbert spaces represents a potentially serious issue for AdS/CFT.

Indeed, the most direct generalization of the reconstruction theorem from Ref. [15] to approximate relative entropy equalities does not lead to the goal of full approximate quantum-error correction but rather to a significantly weaker structure known as a zero-bit code $[20,21]$. Ignoring this distinction can lead to qualitatively wrong conclusions for code spaces whose dimension grows too fast in the large- $N$ limit (e.g., code spaces containing a large number of black-hole microstates) [22]. For mixed states in such code spaces, the entanglement wedge of a boundary region $A$ may be strictly smaller than the bulk complement of the entanglement wedge of the complementary boundary region $\bar{A}$ even in the limit $N \rightarrow \infty$. A naive application of Ref. [15] would incorrectly suggest that any operator in the larger latter region can be reconstructed in region $A$; in fact, only operators in the entanglement wedge of $A$ can be reconstructed in a stateindependent way.

In this article, we demonstrate the entanglement wedge reconstruction conjecture without assuming exact equality of bulk and boundary relative entropies. In doing so, we also provide an explicit formula for entanglement wedge reconstruction. Our analysis builds on recent results in quantum-information theory giving sufficient conditions to approximately reverse the effects of noise. We show that having access only to a subregion of the boundary is equivalent to throwing away or damaging a known part of the error-correcting code describing the bulk spacetime, and it is this correspondence which opens the door to the methods of universal recovery channels. A general quantum map known as a quantum channel $\mathcal{N}$ (i.e., a completely positive, trace-preserving map) is said to be reversible if there exists another quantum channel $\mathcal{R}$ known as the recovery channel, such that the composition $\mathcal{R} \circ \mathcal{N}$ acts as the identity on all states in the domain of $\mathcal{N}$ (i.e., $\mathcal{R} \circ \mathcal{N}[\rho]=\rho$ ). For example, all unitary operations are reversible, with the adjoint of the unitary acting as a recovery channel (since $U^{\dagger} U=\mathbb{1}$ ), and quantum-errorcorrecting codes are designed around noise processes such that the noise can be reversed on the code subspace.

When a channel $\mathcal{N}$ is reversible, it has been known for some time how to construct a recovery channel $\mathcal{R}$ [23]. Exact reversibility will almost never be satisfied in practice, however, and for many applications one may require only that a channel be approximately reversible. For example, in 
approximate quantum-error correction, one requires only that the recovered state $\mathcal{R} \circ \mathcal{N}[\rho]$ be close to the input $\rho$ up to some small tolerance. For a channel that is not exactly reversible, it is natural to ask whether or not there exists a recovery channel that works approximately in the above sense [24]. This question has spurred a flurry of research and was answered only recently in Ref. [25], wherein it was shown that for any quantum channel $\mathcal{N}$, there indeed exists an approximate recovery channel $\mathcal{R}$ such that $\mathcal{R} \circ \mathcal{N}[\rho] \approx \rho, \forall \rho$, with the quality of the approximation controlled by the behavior of the relative entropy under the action of the channel $\mathcal{N}$. We review these recent results in the next section. In the context of AdS/CFT, there is a map from the bulk to the boundary, and a noisy quantum channel arises from tracing over a subregion of the boundary. Our ultimate goal is to recover from that noise process (i.e., recover from the loss of part of the boundary).

The paper is organized as follows. We begin with a review of recent results on universal recovery channels, as well as a few basics of AdS/CFT and recent results in holography. We then apply the theory of universal recovery channels to the problem of entanglement wedge reconstruction in AdS/CFT, and we arrive at an explicit expression for a bulk operator recovered on the boundary. After discussing its salient structural properties, we sketch how our formula applies to $\mathrm{AdS}_{3}$-Rindler reconstruction, and we conclude with possible avenues for future research. In the Appendixes, we prove our entanglement wedge reconstruction result for arbitrary finite-dimensional algebras of observables, thereby obviating simplifying assumptions used in the main body of the paper. To do this, we extend the universal recovery results of Ref. [25] to finitedimensional von Neumann algebras. Importantly, we also prove that approximate recovery channels automatically approximately preserve the multiplicative structure of the original bulk algebra, which ensures that correlation functions of boundary reconstructions of the individual operators, even if each operator is reconstructed using a different entanglement wedge.

\section{BACKGROUND AND PRELIMINARIES}

In this section, we review the theory of recovery channels and basics of AdS/CFT, as well as recent developments in each that we later use to establish our results.

\section{A. Universal recovery channels}

To develop intuition of quantum recovery channels, it is helpful to first set aside quantum mechanics and consider good old probability theory. Our goal is to reverse the effects of applying a stochastic map. In particular, given a stochastic map $p(y \mid x)$ and an observation of $y$, try to infer $x$. One way to do this is to introduce a new stochastic map $p(x \mid y)$ via Bayes's rule

$$
p(x \mid y)=\frac{p(x) p(y \mid x)}{p(y)}
$$

which has the property that $\sum_{y} p\left(x^{\prime} \mid y\right) p(y \mid x)=\delta_{x x^{\prime}}$ if the noise can be reversed. In situations where the noise cannot be perfectly reversed, Bayes's rule provides an excellent (and in many ways optimal) estimate of the input. Since it will prove useful in solving the quantum version of the problem, it is worth noting that we can trivially rewrite Eq. (1) as

$$
p(x \mid y)=\left.\frac{d}{d t}\right|_{t=0} \log \left(\frac{p(y)}{p(x)}+t p(y \mid x)\right) .
$$

In other words, the recovery channel $p(x \mid y)$ can be expressed as the logarithmic directional derivative of the matrix $p(y) p(x)^{-1}$ in the direction of the channel $p(y \mid x)$.

Let us now consider a noncommutative generalization of Bayes's rule. We want to find a quantum channel (i.e., a completely positive, trace-preserving map) that reverses the action of some input channel. Instead of a classical stochastic map, our goal is to reverse the action of a noisy quantum channel. To make the problem precise, consider two Hilbert spaces $\mathcal{H}_{A}$ and $\mathcal{H}_{B}$. Let $S\left(\mathcal{H}_{A}\right)$ and $S\left(\mathcal{H}_{B}\right)$ represent the sets of density operators on systems $A$ and $B$, respectively. A quantum channel $\mathcal{N}: S\left(\mathcal{H}_{A}\right) \rightarrow S\left(\mathcal{H}_{B}\right)$ is said to be reversible if there exists a quantum channel $\mathcal{R}: S\left(\mathcal{H}_{B}\right) \rightarrow S\left(\mathcal{H}_{A}\right)$ called the recovery channel, such that

$$
(\mathcal{R} \circ \mathcal{N})[\rho]=\rho \quad \text { for all } \rho \in S\left(\mathcal{H}_{A}\right) .
$$

A simple example in which reversible channels play a starring role is quantum-error correction. In this case, $\mathcal{N}$ is the composition of encoding some d.o.f. in a code space $\mathcal{H}_{A}$ into a potentially larger Hilbert space, followed by a noise channel (wherein we lose certain d.o.f. or otherwise corrupt the encoded state). $\mathcal{R}$ is then a decoding map, and Eq. (3) corresponds to perfect quantum-error correction.

One way to quantify the noisiness of a quantum channel is by comparing the distinguishability of input states and output states using the relative entropy. Under the action of a quantum channel $\mathcal{N}$, the relative entropy between two states can never increase. This fact is known as the monotonicity of relative entropy or the data processing inequality:

$$
D(\rho \| \sigma) \geq D(\mathcal{N}[\rho] \| \mathcal{N}[\sigma]),
$$

where $D(\rho \| \sigma):=\operatorname{Tr} \rho \log \rho-\operatorname{Tr} \rho \log \sigma$ is the relative entropy between $\rho, \sigma$. If there were a recovery channel $\mathcal{R}$ such that $(\mathcal{R} \circ \mathcal{N})(\rho)=\rho$ and $(\mathcal{R} \circ \mathcal{N})(\sigma)=\sigma$, monotonicity applied a second time to $\mathcal{R}$ would imply saturation of Eq. (4). In fact, the converse is also true. Equality in Eq. (4) holds if and only if there exists a recovery channel $\mathcal{R}$, such that $(\mathcal{R} \circ \mathcal{N})(\rho)=\rho$ and $(\mathcal{R} \circ \mathcal{N})(\sigma)=\sigma$. In this 
case, Petz and co-worker [26] identified an exact recovery channel $\mathcal{R}=\mathcal{P}_{\sigma, \mathcal{N}}$ given by

$$
\mathcal{P}_{\sigma, \mathcal{N}}=\sigma^{1 / 2} \mathcal{N}^{*}\left(\mathcal{N}[\sigma]^{-1 / 2}(\cdot) \mathcal{N}[\sigma]^{-1 / 2}\right) \sigma^{1 / 2},
$$

where $\mathcal{N}^{*}$ denotes the adjoint of the channel $\mathcal{N}$. Because $\mathcal{P}_{\sigma, \mathcal{N}}$ does not depend on $\rho$, it can be used for all $\rho$ saturating Eq. (4) [provided that $\sigma$ is chosen to be full rank to ensure that the relative entropies in Eq. (4) are finite for all states $\rho$ ]. $\mathcal{P}$ is an exact recovery channel often referred to as the Petz map.

Since the condition is necessary and sufficient, failure to saturate Eq. (4) means that an exact recovery map $\mathcal{R}$ cannot exist. But what if $\mathcal{N}$ almost saturates Eq. (4)? In this case, does there exist an approximate recovery map, which would behave well in cases of near saturation, as does Bayes's rule in the stochastic case? Indeed, an approximate version of the recovery channel was developed by Junge et al. [25], who show that, for any $\rho, \sigma \in S\left(\mathcal{H}_{A}\right)$ and any quantum channel $\mathcal{N}$, there exists a recovery channel $\mathcal{R}_{\sigma, \mathcal{N}}$, such that

$$
D(\rho \| \sigma)-D(\mathcal{N}[\rho] \| \mathcal{N}[\sigma]) \geq-2 \log F\left(\rho, \mathcal{R}_{\sigma, \mathcal{N}} \circ \mathcal{N}[\rho]\right),
$$

where $F(\rho, \sigma):=\|\sqrt{\rho} \sqrt{\sigma}\|_{1}$ is the fidelity. The inequality says that the fidelity between the recovered state and the original is controlled by the extent to which the channel saturates monotonicity of relative entropy [Eq. (4)], with perfect fidelity in the case of saturation. Importantly, there is no dependence on the dimension of the Hilbert space. Moreover, Junge et al. [25] gave a concrete expression for the channel $\mathcal{R}_{\sigma, \mathcal{N}}$ called the twirled Petz map and given by

$$
\begin{aligned}
\mathcal{R}_{\sigma, \mathcal{N}}:= & \int_{\mathbb{R}} d t \beta_{0}(t) \sigma^{-[(i t) / 2]} \\
& \times \mathcal{P}_{\sigma, \mathcal{N}}\left(\mathcal{N}[\sigma]^{[(i t) / 2]}(\cdot) \mathcal{N}[\sigma]^{-[(i t) / 2]}\right) \sigma^{[(i t) / 2]},
\end{aligned}
$$

where $\mathcal{P}_{\sigma, \mathcal{N}}$ is the Petz map of Eq. (5), and $\beta_{0}$ is the probability density $\beta_{0}(t):=(\pi / 2)[\cosh (\pi t)+1]^{-1}$. The twirled Petz map is an example of a universal recovery channel, since Eq. (6) holds for any channel $\mathcal{N}$. When $\sigma$ is full rank, both the Petz map and the twirled Petz map are trace-preserving completely positive maps (i.e., quantum channels).

For a completely positive map $\mathcal{N}$, the Choi operator is defined by $\Phi_{\mathcal{N}}:=(\mathrm{id} \otimes \mathcal{N})[\Phi]$, where $|\Phi\rangle=\sum_{j}|j\rangle|j\rangle$ is an un-normalized maximally entangled state. By working with the Choi operator, we can rewrite the recovery channel $\mathcal{R}_{\sigma, \mathcal{N}}$ in a form similar to Eq. (2). In the case of the recovery channel $\mathcal{R}_{\sigma, \mathcal{N}}$, the Choi operator can be expressed as

$$
\Phi_{\mathcal{R}_{\sigma, \mathcal{N}}}=\left.\frac{d}{d t}\right|_{t=0} \log \left(\overline{\mathcal{N}[\sigma]} \otimes \sigma^{-1}+t \Phi_{\mathcal{N}^{*}}\right),
$$

where $\overline{\mathcal{N}}[\sigma]$ is the complex conjugate of $\mathcal{N}[\sigma]$, and $\sigma^{-1}$ is the inverse of $\sigma$ on its support. A proof can be found in the Appendix B. This is the appropriate generalization of Bayes's rule to the noncommutative case. When the channel is reversible, the twirled Petz map $\mathcal{R}_{\sigma, \mathcal{N}}$ reduces to the Petz map $\mathcal{P}_{\sigma, \mathcal{N}}$. In the classical case, both the Petz map and the twirled Petz map reduce to Bayes's rule.

\section{B. AdS/CFT background}

The AdS/CFT correspondence states that quantum gravity in $d+1$ spatial dimensions is dual to a CFT in $d$ spatial dimensions. There are two main dictionaries that describe the mapping between bulk and boundary quantities in the AdS/CFT correspondence: the differentiate dictionary $[27,28]$ and the extrapolate dictionary [29]. The differentiate dictionary relies on the equivalence between the partition functions of the bulk and boundary theories $\left(Z_{\mathrm{CFT}}=Z_{\text {grav }}\right)$. On the other hand, the extrapolate dictionary relies on the fact that local CFT operators living in the boundary theory can be expressed as the limit of appropriately weighted bulk fields as they are taken to the conformal boundary of the AdS spacetime. In particular,

$$
\mathcal{O}(x)=\lim _{z \rightarrow \infty} z^{-\Delta} \phi(x, z),
$$

where $\mathcal{O}$ is a boundary field, $\Delta$ is the scaling dimension of $\mathcal{O}$, and $\phi$ is a bulk field. With this equivalence, boundary correlation functions can be expressed as

$$
\left\langle\mathcal{O}\left(x_{1}\right), \ldots, \mathcal{O}\left(x_{n}\right)\right\rangle_{\mathrm{CFT}}=\lim _{z \rightarrow \infty} z^{-n \Delta}\left\langle\phi\left(x_{1}, z\right), \ldots, \phi\left(x_{n}, z\right)\right\rangle_{\text {bulk }} .
$$

The HKLL procedure [2] uses the extrapolate dictionary and the bulk equations of motion to write a local bulk field operator $\phi(x, z)$ as a smearing of boundary operators $\mathcal{O}$ acting on the "strip" of boundary points that are spacelike separated from $(x, z)$, as shown in Fig. 1. In particular, (a)

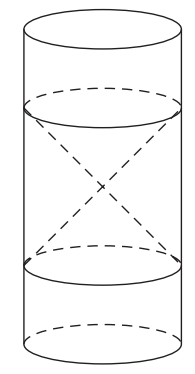

(b)

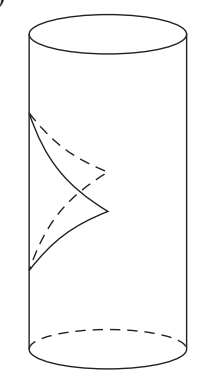

FIG. 1. (a) The HKLL procedure provides a way of writing bulk operators in terms of boundary operators living on a strip in the boundary consisting of all points that are spacelike separated from the bulk point. (b) The causal wedge HKLL procedure provides a way of expressing bulk operators in terms of boundary operators living only in the domain of dependence of a boundary region whose associated causal wedge contains the bulk point. 


$$
\phi(x, z)=\int_{\text {strip }} d x^{\prime} K\left(x, z, x^{\prime}\right) \mathcal{O}\left(x^{\prime}\right)
$$

where the smearing function $K$ can be computed using a mode-sum expansion. The choice of smearing function $K$ is not unique, and there are different choices of $K$ that will reproduce the same bulk field $\phi$. For instance, bulk field operators $\phi(x, z)$ can be written using a smearing function that is supported only on a subset of the boundary, namely, the domain of dependence of a boundary subregion whose causal wedge contains the bulk point, as shown in Fig. 1.

Using the HKLL procedure, one can find representations of a given bulk operator on different regions in the boundary. As observed in Ref. [30], this redundancy is a reflection of the quantum-error-correcting properties of AdS/CFTíf we fix a boundary region $A$, the entanglement wedge of $A$ is defined to be the bulk domain of dependence of any achronal bulk surface bounded by $A$ and the covariant RT minimal surface associated to $A$. For readers unfamiliar with the entanglement wedge, it is easily visualized on a fixed time slice: The entanglement wedge is the region in the bulk bounded by $A$ and the RT surface of $A$. The full spacetime picture of the entanglement wedge is then the bulk domain of dependence of the region on a fixed time slice. The entanglement wedge reconstruction proposal asserts that any local bulk operator acting on the entanglement wedge of $A$ has a representation with support only on $A$.

The key input from holography is a result by Jafferis, Lewkowycz, Maldacena, and Suh (JLMS for short) in which they show that bulk and boundary relative entropies are approximately equal [16]. The relative entropy between two "nice" states in the bulk and their associated states on the boundary are equal to leading order in $1 / N$. To be precise, let $\rho$ and $\sigma$ be two bulk states with the same semiclassical geometry, $\rho_{a}$ and $\sigma_{a}$ be their reduced density matrices on the entanglement wedge of $A, \tilde{\rho}$ and $\tilde{\sigma}$ be the corresponding boundary states, and $\tilde{\rho}_{A}$ and $\tilde{\sigma}_{A}$ be their reduced density matrices on region $A$. Jafferis et al. [16] then showed that

$$
D\left(\tilde{\rho}_{A} \| \tilde{\sigma}_{A}\right)=D\left(\rho_{a} \| \sigma_{a}\right)+\mathcal{O}(1 / N) .
$$

At higher orders in $1 / N$, the equivalence in Eq. (9) is no longer well defined, since the choice of minimal surface used to define the entanglement wedge becomes state dependent. Crucially, AdS/CFT provides only a global map from bulk to boundary states $(\rho \mapsto \tilde{\rho})$. Our approach to entanglement wedge reconstruction will be to construct a suitable local quantum channel mapping states in the entanglement wedge to states in the boundary region $\left(\rho_{a} \mapsto \tilde{\rho}_{A}\right)$. Only then can we interpret Eq. (9) as an approximate saturation of the monotonicity of the relative entropy for a quantum channel, so that Eq. (6) guarantees the existence of an approximate recovery map. It is the adjoint of this recovery map that we ultimately use for reconstruction.

\section{ENTANGLEMENT WEDGE RECONSTRUCTION}

We make the following assumptions:

(1) The bulk Hilbert space contains a code subspace that is mapped via a quantum channel into the CFT Hilbert space.

(2) The JLMS relative entropy condition holds to leading order in $1 / N$.

In fact, the first condition is slightly relaxed in the most general version of our result (Theorem 4). Just for the purposes of illustration, we also pretend that the bulk and boundary Hilbert spaces admit simple tensor factorizations, since this is a familiar convention in the community. However, the reader is cautioned that this convention is not actually correct-even for free theories, the Hilbert space does not factorize, and the problem is only compounded for gauge theories. The proper approach is to work at the level of algebras of observables, without ever making a tensor product assumption. As such, we adopt the more general algebraic approach in the Appendix, proving all claims made in this paper rigorously at the level of finitedimensional von Neumann algebras.

With these assumptions, let us formalize the problem. Let $\mathcal{H}_{\text {code }}$ be a code space with density operators $S\left(\mathcal{H}_{\text {code }}\right)$, and let $\mathcal{H}_{\mathrm{CFT}}$ be the Hilbert space of a CFT with density operators $S\left(\mathcal{H}_{\mathrm{CFT}}\right)$. There are many valid definitions of what it means to be a code space in AdS/CFT, but our results hold for any suitable definition. For instance, one can define the code space to be the set of all states formed by acting with a finite number of low-energy local bulk operators on the vacuum [15,30]. In this context, low energy means the action of the operator does not change the bulk geometry appreciably. The AdS/CFT correspondence relates states in $S\left(\mathcal{H}_{\text {code }}\right)$ to states in $S\left(\mathcal{H}_{\mathrm{CFT}}\right)$. We model this relationship by an isometry $J: \mathcal{H}_{\text {code }} \rightarrow \mathcal{H}_{\mathrm{CFT}}$ embedding the code space into the CFT Hilbert space. Note that $\mathcal{H}_{\text {code }}$ can be identified with its image under the isometry $J$, resulting in the code subspace of previous works [15,31]. In general, one could consider an arbitrary quantum channel mapping states on the code space to states on the CFT Hilbert space. We prove our general result in Theorem 4 without requiring the mapping between code and CFT states to be an isometry.

We now partition the CFT into two regions $A$ and $\bar{A}$, and we partition the bulk into $a$ and $\bar{a}$, where $a$ is supported only on the entanglement wedge of $A$, as shown in Fig. 2. As discussed earlier, we assume that $\mathcal{H}_{\mathrm{CFT}}=\mathcal{H}_{A} \otimes \mathcal{H}_{\bar{A}}$ and that $\mathcal{H}_{\text {code }}=\mathcal{H}_{a} \otimes \mathcal{H}_{\bar{a}}$. The problem of entanglement wedge reconstruction then amounts to constructing a boundary observable $\mathcal{O}_{A}$ supported only on $A$, such that, for any bulk operator $\phi_{a}$ supported in the entanglement wedge $a$ of $A$, 
(a)

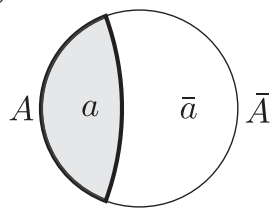

(b)

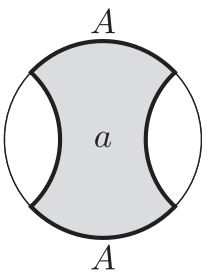

FIG. 2. (a) A bipartition of the boundary into a connected piece $A$ and its complement $\bar{A}$. In this case, the entanglement wedge of $A$ coincides with the causal wedge of $A$ labeled by $a$ in the figure. $\bar{a}$ is then the complement of $a$. (b) A bipartition of the boundary into $A$ and $\bar{A}$ such that $A$ consists of two disconnected components. In the figure above, $A$ spans just more than half of the boundary, and in this case, the entanglement wedge of $A$ is not simply the union of the causal wedges of each piece of $A$. In the bulk, $a$ represents the entanglement wedge of $A$ and $\bar{a}$ is the complement of $a$.

$$
\left|\left\langle\mathcal{O}_{A}\right\rangle_{J \rho J^{\dagger}}-\left\langle\phi_{a}\right\rangle_{\rho}\right| \leq \delta\left\|\phi_{a}\right\|
$$

for all $\rho \in S\left(\mathcal{H}_{\text {code }}\right)$ and for some small $\delta>0$. Using our new notation, Eq. (9) says that, for all $\rho, \sigma \in S\left(\mathcal{H}_{\text {code }}\right)$,

$$
\left|D\left(\rho_{a} \| \sigma_{a}\right)-D\left(\left(J \rho J^{\dagger}\right)_{A} \|\left(J \sigma J^{\dagger}\right)_{A}\right)\right| \leq \epsilon,
$$

where $\epsilon$ is controlled by $1 / N$, and the notation $(\cdot)_{A}:=$ $\operatorname{Tr}_{\bar{A}}(\cdot)$ is shorthand we use throughout.

Note that in this paper, we use the approximate equality of relative entropies in $a$ and $A$ in order to prove that operators in region $a$ can be reconstructed in $A$. In contrast, the starting assumption in Ref. [15] was an exact equality between relative entropies in $\bar{a}$ and $\bar{A}$. As we discuss in the Introduction, an approximate version of this latter condition implies only that the zero bits of region $a$ are encoded in region $A$, which is a significantly weaker condition than full entanglement wedge reconstruction [20-22].

Since the relative entropies approximately agree, one might expect that we can find a universal recovery channel that would undo the effect of the partial trace over $\bar{A}$. However, there is an obstacle: A priori, $\left(J \rho J^{\dagger}\right)_{A}$ depends on the state $\rho$ defined on the whole bulk not just on the reduced state on the entanglement wedge $\rho_{a}$. In this form, the theory of recovery channels is not applicable. To overcome this challenge, we first restrict the recovery problem to special code states of the form $\rho=\rho_{a} \otimes \sigma_{\bar{a}}$, where $\sigma_{\bar{a}}$ is some fixed fiducial state. We thus obtain a quantum channel $\rho_{a} \mapsto\left(J \rho J^{\dagger}\right)_{A}$ mapping states on the entanglement wedge to states on the boundary region. We then verify that the recovery map $\mathcal{R}$ obtained for this channel works in fact for all code states $\rho$, only increasing the error by a small amount, since Eq. (11) also implies that the CFT states corresponding to any $\rho$ and its factorized version $\rho_{a} \otimes \sigma_{\bar{a}}$ are approximately indistinguishable on the boundary region $A$. The adjoint $\mathcal{R}^{*}$ of the recovery channel then

maps bulk operators $\phi_{a}$ supported in the entanglement wedge to boundary operators $\mathcal{O}_{A}$ supported on $A$ and satisfying Eq. (10), thereby achieving entanglement wedge reconstruction.

In more mathematical detail, we first define the local channel $\mathcal{N}: S\left(\mathcal{H}_{a}\right) \rightarrow S\left(\mathcal{H}_{A}\right)$ by

$$
\mathcal{N}\left[\rho_{a}\right]:=\operatorname{Tr}_{\bar{A}}\left[J\left(\rho_{a} \otimes \sigma_{\bar{a}}\right) J^{\dagger}\right]=\left(J\left(\rho_{a} \otimes \sigma_{\bar{a}}\right) J^{\dagger}\right)_{A}
$$

for all states $\rho_{a} \in S\left(\mathcal{H}_{a}\right)$, where $\sigma_{\bar{a}}$ is some fixed full-rank state. If we also choose a full-rank $\sigma_{a} \in S\left(\mathcal{H}_{a}\right)$, then we can use Eq. (7) to obtain a recovery channel $\mathcal{R}=\mathcal{R}_{\sigma_{a}, \mathcal{N}}$ such that, for all $\rho_{a} \in S\left(\mathcal{H}_{a}\right)$,

$$
-2 \log F\left(\rho_{a}, \mathcal{R} \circ \mathcal{N}\left[\rho_{a}\right]\right) \leq\left|D\left(\rho_{a} \| \sigma_{a}\right)-D\left(\mathcal{N}\left[\rho_{a}\right] \| \mathcal{N}\left[\sigma_{a}\right]\right)\right| .
$$

However, by Eq. (11), we have

$$
\left|D\left(\rho_{a} \| \sigma_{a}\right)-D\left(\mathcal{N}\left[\rho_{a}\right] \| \mathcal{N}\left[\sigma_{a}\right]\right)\right| \leq \epsilon,
$$

and therefore, we conclude that the recovery channel $\mathcal{R}$ works with high fidelity (cf. Ref. [25] Corollary 6.1). By one of the Fuchs-van de Graaf inequalities [32], we have that

$$
\left\|\rho_{a}-\mathcal{R}\left(\mathcal{N}\left[\rho_{a}\right]\right)\right\|_{1} \leq 2 \sqrt{\epsilon}:=\delta_{1}
$$

for all $\rho_{a} \in S\left(\mathcal{H}_{a}\right)$. We now show that the channel $\mathcal{R}$ recovers the reduced state on the entanglement wedge for arbitrary code states $\rho$, not just for those of the form $\rho=\rho_{a} \otimes \sigma_{\bar{a}}$ :

$$
\begin{aligned}
\left\|\mathcal{N}\left[\rho_{a}\right]-\left(J \rho J^{\dagger}\right)_{A}\right\|_{1}^{2} & =\left\|\left[J\left(\rho_{a} \otimes \sigma_{\bar{a}}\right) J^{\dagger}\right]_{A}-\left(J \rho J^{\dagger}\right)_{A}\right\|_{1}^{2} \\
& \leq 2 \ln 2 D\left(\left(J\left(\rho_{a} \otimes \sigma_{\bar{a}}\right) J^{\dagger}\right)_{A} \|\left(J \rho J^{\dagger}\right)_{A}\right) \\
& \leq(2 \ln 2) \epsilon=: \delta_{2}^{2},
\end{aligned}
$$

where the first inequality is Pinsker's inequality, and the second inequality is Eq. (11), with one state set to $\rho$ and the other set to $\rho_{a} \otimes \sigma_{\bar{a}}$. Therefore, we obtain that, for all $\rho \in S\left(\mathcal{H}_{\text {code }}\right)$,

$$
\begin{aligned}
\| \rho_{a} & -\mathcal{R}\left[\left(J \rho J^{\dagger}\right)_{A}\right] \|_{1} \\
& \leq\left\|\rho_{a}-\mathcal{R}\left(\mathcal{N}\left[\rho_{a}\right]\right)\right\|_{1}+\left\|\mathcal{R}\left(\mathcal{N}\left[\rho_{a}\right]\right)-\mathcal{R}\left[\left(J \rho J^{\dagger}\right)_{A}\right]\right\|_{1} \\
& \leq\left\|\rho_{a}-\mathcal{R}\left(\mathcal{N}\left[\rho_{a}\right]\right)\right\|_{1}+\left\|\mathcal{N}\left[\rho_{a}\right]-\left(J \rho J^{\dagger}\right)_{A}\right\|_{1} \\
& \leq \delta_{1}+\delta_{2}=: \delta
\end{aligned}
$$

Thus, we conclude that $\mathcal{R}$ recovers arbitrary bulk states in the entanglement wedge with high fidelity, as desired. We now show that the adjoint of the map $\mathcal{R}$ solves the entanglement wedge reconstruction problem in the form of Eq. (10). Given a bulk operator $\phi_{a}$ supported in entanglement wedge $a$ of $A$, define $\mathcal{O}_{A}=\mathcal{R}^{*}\left[\phi_{a}\right]$. Then we have that, for all $\rho \in S\left(\mathcal{H}_{\text {code }}\right)$, 


$$
\begin{aligned}
\left|\left\langle\mathcal{O}_{A}\right\rangle_{J \rho J^{\dagger}}-\left\langle\phi_{a}\right\rangle_{\rho}\right| & =\left|\operatorname{Tr} \mathcal{R}^{*}\left[\phi_{a}\right]\left(J \rho J^{\dagger}\right)_{A}-\operatorname{Tr} \phi_{a} \rho_{a}\right| \\
& =\left|\operatorname{Tr} \phi_{a} \mathcal{R}\left[\left(J \rho J^{\dagger}\right)_{A}\right]-\operatorname{Tr} \phi_{a} \rho_{a}\right| \\
& =\left|\operatorname{Tr} \phi_{a}\left(\mathcal{R}\left[\left(J \rho J^{\dagger}\right)_{A}\right]-\rho_{a}\right)\right| \\
& \leq\left\|\mathcal{R}\left[\left(J \rho J^{\dagger}\right)_{A}\right]-\rho_{a}\right\|_{1}\left\|\phi_{a}\right\| \leq \delta\left\|\phi_{a}\right\|,
\end{aligned}
$$

where the first inequality is Hölder's inequality and the second is Eq. (13). Thus, we have arrived at the desired approximate equality of one-point functions.

\section{CORRELATION FUNCTIONS}

Given a set of $n$ bulk operators $\left\{\phi_{a}^{(i)}\right\}$ acting on the entanglement wedge $a$ of $A$, our result implies that we can calculate their $n$-point correlation function as the expectation value of the boundary operator $O_{A}=\mathcal{R}^{*}\left[\prod_{i} \phi_{a}^{(i)}\right]$ obtained by reconstructing the composite bulk operator $\prod_{i} \phi_{a}^{(i)}$. However, one might hope that the reconstructed operators approximately reproduce the bulk algebra in the sense that

$$
\left\langle\prod_{i} \phi_{a}^{(i)}\right\rangle_{\rho} \approx\left\langle\prod_{i} \mathcal{O}_{A}^{(i)}\right\rangle_{J \rho J^{\dagger}}
$$

where $\mathcal{O}_{A}^{(i)}=\mathcal{R}^{*}\left(\phi_{a}^{(i)}\right)$. When the bulk and boundary relative entropies are exactly equal, it is known that $\mathcal{R}^{*}$ is an algebra homomorphism (Ref. [26] Proposition 8.4), so that Eq. (14) holds with equality. We prove in Theorem 4 in the Appendix that when the bulk and boundary relative entropies are only approximately equal, as in Eq. (11), then Eq. (14) still holds approximately, although the size of the error may grow quadratically with $n$.

Furthermore, we show in Corollary 5 that the above result continues to be true even when different operators are reconstructed using the entanglement wedges $a_{i}$ of different boundary regions $A_{i}$ :

$$
\left\langle\prod_{i} \phi_{a_{i}}^{(i)}\right\rangle_{\rho} \approx\left\langle\prod_{i} \mathcal{O}_{A_{i}}^{(i)}\right\rangle_{J \rho J^{\dagger}},
$$

where $\mathcal{O}_{A_{i}}^{(i)}=\mathcal{R}_{A_{i}}^{*}\left[\phi_{a_{i}}^{(i)}\right]$, and $\mathcal{R}_{A_{i}}$ is the recovery map for boundary region $A_{i}$. This result is illustrated in Fig. 3.

\section{AN EXPLICIT FORMULA}

Our ultimate goal is an explicit formula for approximate entanglement wedge reconstruction. Thus, we want to calculate $\mathcal{O}_{A}=\mathcal{R}^{*}\left[\phi_{a}\right]$ explicitly by using Eq. (7). Recall that the recovery channel $\mathcal{R}$ depends on our choice of $\sigma_{a}$ and, through the channel $\mathcal{N}$ from Eq. (12), also on the choice of $\sigma_{\bar{a}}$. The result is particularly satisfying when both $\sigma_{a}$ and $\sigma_{\bar{a}}$ are chosen to be maximally mixed. It is important to emphasize that this is just a convenient choice that we make in order to simplify our expressions. For an infinite-dimensional code space, such a choice would not be well defined, and instead another choice of average

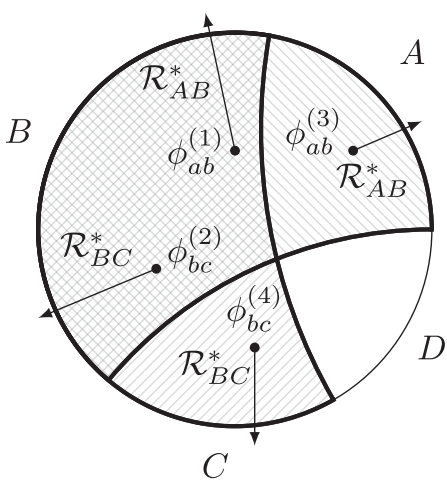

FIG. 3. We show in Eq. (15) that correlation functions of bulk operators can be computed by pushing each bulk operator to the boundary separately and computing the expectation value in the boundary theory. The bulk operators need not live in the same entanglement wedge. In this figure, the boundary is decomposed into four regions: $A, B, C$, and $D$. Regions $A B$ and $B C$ have a nontrivial intersection, and the bulk operators $\phi$ are localized to the regions as shown. We can use the recovery maps $\mathcal{R}_{A B}^{*}$ and $\mathcal{R}_{B C}^{*}$ to push the operators to $A B$ and $B C$, respectively.

code state can be used. With this simplification, we find that, for all bulk operators $\phi_{a}$ with support in the entanglement wedge $a$,

$$
\begin{aligned}
\mathcal{O}_{A} & :=\mathcal{R}^{*}\left[\phi_{a}\right] \\
& =\frac{1}{d_{\text {code }}} \int_{\mathbb{R}} d t \beta_{0}(t) e^{\frac{1}{2}(1-i t) H_{A}} \operatorname{Tr}_{\bar{A}}\left[J\left(\phi_{a} \otimes \mathbb{1}_{\bar{a}}\right) J^{\dagger}\right] e^{\frac{1}{2}(1+i t) H_{A}},
\end{aligned}
$$

where $H_{A}=-\log \left(J \tau J^{\dagger}\right)_{A}$ is the boundary modular Hamiltonian on subregion $A$ associated with the maximally mixed state $\tau$ on the code subspace. The above expression makes it clear that the natural basis one should use for entanglement wedge reconstruction is the eigenbasis of the modular Hamiltonian. What is more, the recovery channel can be expressed in the form of a logarithmic directional derivative, as in Eq. (8):

$\mathcal{O}_{A}=\mathcal{R}^{*}\left[\phi_{a}\right]=-\left.\frac{1}{d_{\text {code }}} \frac{d}{d t}\right|_{t=0} H_{A}\left[\tau_{\text {code }}+t \phi_{a} \otimes \mathbb{1}_{\bar{a}}\right]$,

where we write $H_{A}[\rho]:=-\log \left(J \rho J^{\dagger}\right)_{A}$ for the boundary modular Hamiltonian on subregion $A$ associated with a bulk state $\rho$. In other words, the boundary operator corresponding to $\phi_{a}$ can be computed as the response in the boundary modular Hamiltonian $H_{A}$ to a perturbation of the maximally mixed code state in the direction of the operator $\phi_{a}$.

Equations (16) and (17) are explicit expressions for entanglement wedge reconstruction that are meaningful even when the bulk and boundary relative entropies are not exactly equal. When the relative entropies are exactly equal for all $\rho$, they reduce to the simple Petz map, which is equivalent to existing notions of operator algebra quantumerror correction, as applied to bulk reconstruction. 
In Eq. (16), we first map our bulk operator $\phi_{a}$ to the entire boundary via $\phi_{a} \mapsto J\left(\phi_{a} \otimes \mathbb{1}_{\bar{a}}\right) J^{\dagger}$. One might wonder what the connection is between this mapping and the global HKLL reconstruction procedure [2] discussed in the preliminaries, which likewise produces an operator $\mathcal{O}_{\text {HKLL }}$ supported on the full CFT Hilbert space and satisfying $\left\langle\phi_{a}\right\rangle_{\rho}=\left\langle\mathcal{O}_{\mathrm{HKLL}}\right\rangle_{J \rho J^{\dagger}}$. The latter condition means that $J^{\dagger} \mathcal{O}_{\mathrm{HKLL}} J=\phi_{a} \otimes \mathbb{1}_{\bar{a}}$. Hence,

$$
J\left(\phi_{a} \otimes \mathbb{1}_{\bar{a}}\right) J^{\dagger}=J J^{\dagger} \mathcal{O}_{\mathrm{HKLL}} J J^{\dagger} .
$$

The map $J J^{\dagger}$ is precisely the projection onto the code subspace. This means that, in order to compute the term $J\left(\phi_{a} \otimes \mathbb{1}_{\bar{a}}\right) J^{\dagger}$ in our formula (16), we can leverage the global HKLL procedure to map out the bulk operator to a global boundary operator if we make sure to subsequently project the result onto the code subspace. Such a projection onto the code subspace is not necessarily complicated.

In the Appendix, we work through an explicit calculation involving our reconstruction formula. The example is analogous to Rindler wedge reconstruction, although it is only strictly true for free fields. We consider a bulk operator $\phi_{a}$ in $\mathrm{AdS}_{3}$ localized in the entanglement wedge of a boundary interval, and we choose a two-dimensional code space spanned by states $|\tilde{0}\rangle$ and $\phi_{a}|\tilde{0}\rangle$, where $|\tilde{0}\rangle$ is the vacuum state. Using only our recovery formula and global HKLL, we find an expression for reconstructed bulk operators as a mode expansion supported on the Rindler wedge.

\section{DISCUSSION}

The mapping of bulk operators to boundary subregions was recognized as a problem in operator algebra quantumerror correction in Ref. [30]. This important conceptual advance, along with the insight that bulk and boundary relative entropies agree [16], imply that any low-energy bulk operator in the entanglement wedge of a boundary region should be representable as an operator acting on that boundary region $[15,16]$. In this sense, a boundary region is dual to its entanglement wedge.

In this article, we use recent advances in quantuminformation theory to provide a robust demonstration of this result that does not assume that the bulk and boundary relative entropies are exactly equal. In addition, we find a satisfyingly simple explicit formula for the boundary operator, namely, that it can be computed as the response of the boundary modular Hamiltonian of the subregion to a perturbation of the average code state in the direction of the bulk operator.

Our argument does not rely on the structural consequences implied by exact equality of relative entropies assumed in the proof of the entanglement wedge reconstruction conjecture in Ref. [15]. That said, the argument that we present here still assumes the finite dimensionality of the associated von Neumann algebras. Most of our expressions can be applied formally even in the infinite-dimensional setting, but it would be a worthwhile project to try to rigorously extend our results to the infinitedimensional case. It seems likely that additional hypotheses will be required in order to ensure the existence of the local channel that is instrumental in our argument.

In independent work [33], Faulkner and Lewkowycz arrived at a formula for entanglement wedge reconstruction which also involves modular flow. Their approach builds on the insights of Ref. [16] and uses the free-field physics of the bulk to argue that entanglement wedge reconstruction involves integrating the modular flow against a certain (generally unknown) kernel. It would interesting to understand their results from the perspective of our framework; most likely, this requires further exploration of the consequences of the free-field assumption in the bulk which we do not a priori need to assume in our approach.

\section{ACKNOWLEDGMENTS}

We thank Mario Berta, Tom Faulkner, Daniel Harlow, Eliot Hijano, Aitor Lewkowycz, Sepehr Nezami, Jonathan Oppenheim, David Sutter, Mark Van Raamsdonk, and Mark Wilde for helpful discussions and feedback. BGS is supported by the Simons Foundation's It from Qubit Collaboration, through a Simons Investigator grant to Senthil Todadri and by Multidisciplinary University Research Initiatives (MURI) Grant No. W911NF-14-10003 from ARO. G. S. is grateful to Canada's NSERC for a postgraduate scholarship, and to the IQIM at Caltech and the SITP at Stanford. M.W. and P. H. gratefully acknowledge support from the Simons Foundation's Investigator program and It from Qubit Collaboration, as well as AFOSR Grant No. FA9550-16-1-0082. P. H. is also supported by CIFAR. M. W. also acknowledges financial support from the NWO through Veni Grant No. 680-47-459.

Note added in proof.-In follow-up work to this paper, it has also recently been shown that one can perform entanglement wedge reconstruction using only the Petz map as opposed to the twirled Petz map [34].

\section{APPENDIX A: FINITE-DIMENSIONAL VON NEUMANN ALGEBRAS}

A finite-dimensional von Neumann algebra is a (unital) subalgebra $\mathcal{A} \subseteq B(\mathcal{H})$ of the linear operators on some finitedimensional Hilbert space $\mathcal{H}$. We define the set of states as the intersection $S(\mathcal{A})=\mathcal{A} \cap S(\mathcal{H})$ of the algebra with the set of all density operators on the Hilbert space $\mathcal{H}$ denoted by $S(\mathcal{H})$. We denote the larger space of positive linear functionals (with no normalization condition) on $\mathcal{A}$ by $P(\mathcal{A})$. We write $\langle\phi\rangle_{\rho}:=\operatorname{Tr} \rho \phi$ for the expectation value of an operator $\phi \in \mathcal{A}$ in state 
$\rho \in S(\mathcal{A})$. [To any state $\rho \in S(\mathcal{A})$, we may assign the positive normalized linear functional $\phi \mapsto\left\langle\phi_{\rho}\right\rangle$, thereby connecting $S(\mathcal{A})$ with the standard definition of states on a von Neumann algebra.]

In this way, we may lift standard definitions in quantum-information theory to finite-dimensional von Neumann algebras. For example, the trace norm difference $\|\rho-\sigma\|_{1}$, the relative entropy $D(\rho \| \sigma)$, and the fidelity $F(\rho, \sigma)$ for $\rho, \sigma \in S(\mathcal{A})$ can be defined in the usual way by $\|\rho-\sigma\|_{1}:=\operatorname{Tr}|\rho-\sigma|, D(\rho \| \sigma):=\operatorname{Tr}[\rho \log \rho-\rho \log \sigma]$, and $F(\rho, \sigma):=\|\sqrt{\rho} \sqrt{\sigma}\|_{1}$, respectively, in agreement with their abstract definitions for von Neumann algebras. For a completely positive map $\mathcal{N}: P(\mathcal{A}) \rightarrow P(\mathcal{B})$, the adjoint or dual channel $\mathcal{N}^{*}: \mathcal{B} \rightarrow \mathcal{A}$ is defined by demanding that $\langle\phi\rangle_{\mathcal{N}[\rho]}=\left\langle\mathcal{N}^{*}[\phi]\right\rangle_{\rho}$ for all $\rho \in S(\mathcal{A})$ and $\phi \in \mathcal{B}$. If $\mathcal{N}$ is trace preserving, then it is called a quantum channel (equivalently, the dual map is unital, i.e., $\mathcal{N}^{*}[\mathbb{1}]=\mathbb{1}$ ). It is called a quantum operation if $\mathcal{N}$ is merely trace nonincreasing (equivalently, $\mathcal{N}^{*}[\mathbb{1}] \leq \mathbb{1}$ ).

Consider a (unital) subalgebra $\mathcal{A} \subseteq \mathcal{B}$. For any state $\rho \in S(\mathcal{B})$, we define its restriction $\left.\rho\right|_{\mathcal{A}}$ as the unique element in $S(\mathcal{A})$ such that $\langle\phi\rangle_{\left.\rho\right|_{\mathcal{A}}}=\left\langle\phi_{\rho}\right\rangle$ for all $\phi \in \mathcal{A}$; the assignment $\left.\rho \mapsto \rho\right|_{\mathcal{A}}$ defines a quantum channel. The inclusion map $\mathcal{E}_{\mathcal{A}}: S(\mathcal{A}) \subseteq$ $S(\mathcal{B})$ is likewise a quantum channel sometimes referred to as a state extension. (In the language of von Neumann algebras, it is the predual of a conditional expectation onto $\mathcal{A}$.) Importantly,

$$
\left.\mathcal{E}_{\mathcal{A}}[\rho]\right|_{\mathcal{A}}=\rho
$$

for all $\rho \in S(\mathcal{A})$. Since $\mathcal{E}_{\mathcal{A}}$ is just the inclusion map, it is immediate that

$$
\begin{aligned}
D(\rho \| \sigma) & =D\left(\mathcal{E}_{\mathcal{A}}[\rho] \| \mathcal{E}_{\mathcal{A}}[\sigma]\right) \quad \text { and } \\
F(\rho, \sigma) & =F\left(\mathcal{E}_{\mathcal{A}}[\rho], \mathcal{E}_{\mathcal{A}}[\sigma]\right)
\end{aligned}
$$

for all $\rho, \sigma \in S(\mathcal{A})$.

Lastly, we show that there is a natural generalization of Stinespring's dilation theorem to quantum operations on finitedimensional von Neumann algebras.

Lemma 1. Let $\mathcal{N}: P(\mathcal{A}) \rightarrow P(\mathcal{B})$ be a quantum operation on finite-dimensional von Neumann algebras with $\mathcal{A} \subseteq$ $B\left(\mathcal{H}_{A}\right)$ and $\mathcal{B} \subseteq B\left(\mathcal{H}_{B}\right)$. Then,

$$
\mathcal{N}(\rho)=\left.\left[V \mathcal{E}_{A}(\rho) V^{\dagger}\right]\right|_{\mathcal{B}},
$$

where $V: \mathcal{H}_{A} \rightarrow \mathcal{H}_{B} \otimes \mathcal{H}_{E}$ (for some auxiliary Hilbert space $\mathcal{H}_{E}$ ) and $V^{\dagger} V \leq \mathbb{1}$.

Proof.-We define

$$
\tilde{\mathcal{N}}: P\left(\mathcal{H}_{A}\right) \rightarrow P\left(\mathcal{H}_{B}\right), \quad \omega \rightarrow \mathcal{E}_{\mathcal{B}}\left(\mathcal{N}\left[\left.\omega\right|_{\mathcal{A}}\right]\right)
$$

This is a quantum operation between states on Hilbert spaces and hence has a Stinespring dilation $V: \mathcal{H}_{A} \rightarrow \mathcal{H}_{B} \otimes \mathcal{H}_{E}$ such that $\tilde{\mathcal{N}}=\operatorname{Tr}_{E} V(\cdot) V^{\dagger}$. However, it follows from Eq. (A1) that

$$
\mathcal{N}=\left.\left[\tilde{\mathcal{N}} \circ \mathcal{E}_{\mathcal{A}}(\cdot)\right]\right|_{\mathcal{B}}
$$

and thus, we obtain Lemma 1.

We refer to Refs. $[26,31,35]$ for more detailed expositions of the theory of finite-dimensional von Neumann algebras.

\section{APPENDIX B: APPROXIMATE RECOVERY MAPS FOR VON NEUMANN ALGEBRAS}

We now extend the universal recovery result of Ref. [25] to finite-dimensional von Neumann algebras [cf. Eq. (6) herein].

Lemma 2. Let $\mathcal{N}: S(\mathcal{A}) \rightarrow S(\mathcal{B})$ be a quantum channel of finite-dimensional von Neumann algebras and $\rho, \sigma \in S(\mathcal{A})$ states such that $\operatorname{supp} \rho \subseteq \operatorname{supp} \sigma$. Then,

$$
D(\rho \| \sigma)-D(\mathcal{N}[\rho] \| \mathcal{N}[\sigma]) \geq-2 \log F\left(\rho,\left(\mathcal{R}_{\sigma, \mathcal{N}} \circ \mathcal{N}\right)[\rho]\right)
$$

where 


$$
\mathcal{R}_{\sigma, \mathcal{N}}[\gamma]:=\int d t \beta_{0}(t) \sigma^{-i t / 2} \mathcal{P}_{\sigma, \mathcal{N}}\left(\mathcal{N}[\sigma]^{i t / 2} \gamma \mathcal{N}[\sigma]^{-i t / 2}\right) \sigma^{i t / 2}
$$

is a quantum operation defined in terms of the Petz recovery map

$$
\mathcal{P}_{\sigma, \mathcal{N}}[\gamma]=\sigma^{1 / 2} \mathcal{N}^{*}\left(N[\sigma]^{-1 / 2} \gamma \mathcal{N}[\sigma]^{-1 / 2}\right) \sigma^{1 / 2},
$$

and the probability distribution $\beta_{0}(t)=(\pi / 2)[\cosh (\pi t)+1]^{-1}$.

Proof.-By assumption, $\mathcal{A} \subseteq B\left(\mathcal{H}_{A}\right)$ and $\mathcal{B} \subseteq B\left(\mathcal{H}_{B}\right)$ for finite-dimensional Hilbert spaces $\mathcal{H}_{A}, \mathcal{H}_{B}$. We denote by $\mathcal{E}_{\mathcal{A}}$ and $\mathcal{E}_{\mathcal{B}}$ the corresponding state extension maps defined above. As in Lemma 1, we now consider

$$
\tilde{\mathcal{N}}: S\left(\mathcal{H}_{A}\right) \rightarrow S\left(\mathcal{H}_{B}\right), \quad \omega \mapsto \mathcal{E}_{\mathcal{B}}\left(\mathcal{N}\left[\left.\omega\right|_{\mathcal{A}}\right)\right.
$$

This is a quantum channel between density operators on Hilbert spaces, and hence, Ref. [25] Theorem 2.1 is applicable. It states that

$$
D(\omega \| \chi)-D(\tilde{\mathcal{N}}[\omega] \| \tilde{\mathcal{N}}[\chi]) \geq-2 \log F\left(\omega,\left(\mathcal{R}_{\chi, \tilde{\mathcal{N}}} \circ \tilde{\mathcal{N}}\right)[\omega]\right)
$$

for any pair of density operators $\omega, \chi \in S(\mathcal{H})$, such that $\operatorname{supp} \omega \subseteq \operatorname{supp} \chi$. We now make the choice $\omega=\mathcal{E}_{A}[\rho]$ and $\chi=\mathcal{E}_{A}[\sigma]$. Then, using Eqs. (A1) and (A2), it follows that $D(\omega \| \chi)=D(\rho \| \sigma), \quad D(\tilde{\mathcal{N}}[\omega] \| \tilde{\mathcal{N}}[\chi])=$

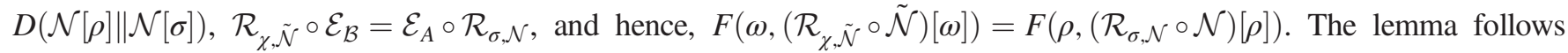
immediately.

If a quantum channel $\mathcal{N}: S(\mathcal{A}) \rightarrow S(\mathcal{B})$ is exactly reversible, then it can be reversed by the Petz recovery map $\mathcal{P}=\mathcal{P}_{\sigma, \mathcal{N}}$ for any faithful state $\sigma$. In this case, $\mathcal{P}^{*}$ is multiplicative in the sense that $\mathcal{P}^{*}\left[\phi^{\prime} \phi\right]=\mathcal{P}^{*}\left[\phi^{\prime}\right] \mathcal{P}^{*}[\phi]$ (e.g., Ref. [26] Proposition 8.4). If $\mathcal{R}$ is an arbitrary quantum operation that reverses $\mathcal{N}$ [i.e., $(\mathcal{R} \circ \mathcal{N})[\rho]=\rho$ for all $\rho$ ], then it is still true that $\left\langle\mathcal{R}^{*}\left[\phi_{1}\right] \mathcal{R}^{*}\left[\phi_{2}\right] \ldots\right\rangle_{\mathcal{N}[\rho]}=\left\langle\mathcal{R}^{*}\left[\phi_{1} \phi_{2} \ldots\right]\right\rangle_{\mathcal{N}[\rho]}=\left\langle\phi_{1} \phi_{2} \ldots\right\rangle_{\rho} \quad$ for $\quad$ all $\rho \in S(\mathcal{A})$, or, equivalently, that $\mathcal{N}^{*}\left(\mathcal{R}^{*}\left[\phi_{1}\right] \mathcal{R}^{*}\left[\phi_{2}\right] \ldots\right)=\mathcal{N}^{*}\left(\mathcal{R}^{*}\left[\phi_{1} \phi_{2} \ldots\right]\right)$. In fact, this is true even when a different (exact) recovery map is used for each operator $\phi_{i}$. We now prove that approximate reversibility implies approximate multiplicativity. Correlation functions are reconstructed up to an error that grows at most quadratically with $n$, even when different recovery maps $\mathcal{R}_{i}$ correcting different subalgebras $\mathcal{A}_{i}$ of the original algebra $\mathcal{A}$ are used for each operator $\phi_{i}$.

Theorem 3. Let $\mathcal{N}: P(\mathcal{A}) \rightarrow P(\mathcal{B})$ and $\mathcal{R}_{i}: P(\mathcal{B}) \rightarrow P\left(\mathcal{A}_{i}\right)$ be quantum operations on finite-dimensional von Neumann algebras, with $\mathcal{A}_{i} \subseteq \mathcal{A}$, and $\epsilon>0$ such that, for each recovery channel $\mathcal{R}_{i}$, we have $\left\|\mathcal{R}_{i} \circ \mathcal{N}[\rho]-\left.\rho\right|_{\mathcal{A}_{i}}\right\|_{1} \leq \epsilon$ for all $\rho \in S(\mathcal{A})$. Then,

$$
\left\|\mathcal{N}^{*}\left(\prod_{i=1}^{n} \mathcal{R}_{i}^{*}\left[\phi_{i}\right]\right)-\prod_{i=1}^{n} \phi_{i}\right\| \leq \frac{1}{2} \epsilon n(3 n-1) \prod_{i=1}^{n}\left\|\phi_{i}\right\|
$$

for all $\phi_{i} \in \mathcal{A}_{i}$.

Proof.-Let $\mathcal{A} \subseteq B\left(\mathcal{H}_{A}\right)$ and $\mathcal{B} \subseteq B\left(\mathcal{H}_{B}\right)$. We define the operators $V: \mathcal{H}_{A} \rightarrow \mathcal{H}_{B} \otimes \mathcal{H}_{E}$ and $W_{i}: \mathcal{H}_{B} \rightarrow \mathcal{H}_{A} \otimes \mathcal{H}_{E^{\prime}}$ to be Stinespring dilations (as in Lemma 1) of $\mathcal{N}=\left.\left[V\left(\mathcal{E}_{\mathcal{A}}(\cdot)\right) V^{\dagger}\right]\right|_{\mathcal{B}}$ and $\mathcal{R}_{i}=\left.\left[W_{i}\left(\mathcal{E}_{\mathcal{B}}(\cdot)\right) W_{i}^{\dagger}\right]\right|_{\mathcal{A}_{i}}$, respectively. Since $\mathcal{N}$ and $\mathcal{R}_{i}$ are quantum operations,

$$
\|V\|,\left\|W_{i}\right\| \leq 1
$$

Let $T_{i}=\sqrt{1-V V^{\dagger}} W_{i}^{\dagger} \phi_{i} W_{i} V$. Then,

$$
\begin{aligned}
0 & \leq T_{i}^{\dagger} T_{i} \leq V^{\dagger} W_{i}^{\dagger} \phi_{i}^{\dagger}\left(\mathbb{1}-W_{i} V V^{\dagger} W_{i}^{\dagger}\right) \phi_{i} W_{i} V \\
& =\mathcal{N}^{*}\left(\mathcal{R}_{i}^{*}\left[\phi_{i}^{\dagger} \phi_{i}\right]\right)-\mathcal{N}^{*}\left(\mathcal{R}_{i}^{*}\left[\phi_{i}\right]\right)^{\dagger} \mathcal{N}^{*}\left(\mathcal{R}^{*}\left[\phi_{i}\right]\right)=\Delta_{1}+\Delta_{2},
\end{aligned}
$$

where $\Delta_{1}:=\mathcal{N}^{*}\left(\mathcal{R}_{i}^{*}\left[\phi_{i}^{\dagger} \phi_{i}\right]\right)-\phi_{i}^{\dagger} \phi_{i}$ and $\Delta_{2}:=\phi_{i}^{\dagger} \phi_{i}-\mathcal{N}^{*}\left(\mathcal{R}_{i}^{*}\left[\phi_{i}\right]\right)^{\dagger} \mathcal{N}^{*}\left(\mathcal{R}_{i}^{*}\left[\phi_{i}\right]\right)$. By assumption,

$$
\left\|\mathcal{N}^{*}\left(\mathcal{R}_{i}^{*}[\chi]\right)-\chi\right\| \leq \epsilon\|\chi\|
$$


for all operators $\chi \in \mathcal{A}_{i}$. Hence, we can bound $\left\|\Delta_{1}\right\| \leq \epsilon\|\phi\|^{2}$ and $\left\|\Delta_{2}\right\| \leq 2 \epsilon\|\phi\|^{2}$, and it follows that

$$
\left\|T_{i}\right\| \leq \sqrt{3 \epsilon}\left\|\phi_{i}\right\|
$$

As a result, we see that

$$
\begin{aligned}
& \left\|V^{\dagger}\left[\prod_{i=1}^{k} W_{i}^{\dagger} \phi_{i} W_{i}\right] \sqrt{\left(1-V V^{\dagger}\right)}\right\| \\
& \leq\left\|V^{\dagger}\left[\prod_{i=1}^{k-1} W_{i}^{\dagger} \phi_{i} W_{i}\right] V V^{\dagger} W_{k}^{\dagger} \phi_{k} W_{k} \sqrt{1-V V^{\dagger}}\right\|+\left\|V^{\dagger}\left[\prod_{i=1}^{k-1} W_{i}^{\dagger} \phi_{i} W_{i}\right]\left(1-V V^{\dagger}\right) W_{k}^{\dagger} \phi_{k} W_{k} \sqrt{1-V V^{\dagger}}\right\| \\
& \quad \leq\left\|T_{k}^{\dagger}\right\| \prod_{i=1}^{k-1}\left\|\phi_{i}\right\|+\left\|V^{\dagger}\left[\prod_{i=1}^{k-1} W_{i}^{\dagger} \phi_{i} W_{i}\right] \sqrt{1-V V^{\dagger}}\right\|\left\|\phi_{k}\right\| \leq \sqrt{3 \epsilon} k \prod_{i=1}^{k}\left\|\phi_{i}\right\|,
\end{aligned}
$$

where the first inequality is the triangle inequality, the second follows from the submultiplicativity of the operator norm, and the last inequality uses induction and Eq. (B3). Hence,

$$
\begin{aligned}
& \left\|V^{\dagger}\left[\prod_{i=1}^{k} W_{i}^{\dagger} \phi_{i} W_{i}\right]\left(1-V V^{\dagger}\right) W_{(k+1)}^{\dagger} \phi_{(k+1)} W_{(k+1)} V \prod_{i=k+2}^{n} V^{\dagger} W_{i}^{\dagger} \phi_{i} W_{i} V\right\| \\
& \leq\left\|V^{\dagger}\left[\prod_{i=1}^{k} W_{i}^{\dagger} \phi_{i} W_{i}\right] \sqrt{\left(1-V V^{\dagger}\right)}\right\|\left\|T_{k+1}\right\| \prod_{i=k+2}^{n}\left\|\phi_{i}\right\| \leq 3 k \epsilon \prod_{i=1}^{n}\left\|\phi_{i}\right\|
\end{aligned}
$$

and

$$
\begin{aligned}
& \left\|\mathcal{N}^{*}\left(\prod_{i=1}^{n} \mathcal{R}_{i}^{*}\left[\phi_{i}\right]\right)-\prod_{i=1}^{n} \mathcal{N}^{*}\left(\mathcal{R}_{i}^{*}\left[\phi_{i}\right]\right)\right\| \\
& =\left\|V^{\dagger}\left[\prod_{i=1}^{n} W_{i}^{\dagger} \phi_{i} W_{i}\right] V-\prod_{i=1}^{n} V^{\dagger} W_{i}^{\dagger} \phi_{i} W_{i} V\right\| \leq \sum_{k=1}^{n-1}\left\|V^{\dagger}\left[\prod_{i=1}^{k} W_{i}^{\dagger} \phi_{i} W_{i}\right]\left(1-V V^{\dagger}\right) W_{(k+1)}^{\dagger} \phi_{k+1} W_{k+1} V\left[\prod_{j=k+2}^{n} V^{\dagger} W_{j}^{\dagger} \phi_{j} W_{j} V\right]\right\| \\
& \leq \frac{3}{2} \epsilon n(n-1) .
\end{aligned}
$$

Finally,

$$
\begin{aligned}
& \left\|\prod_{i=1}^{n} \mathcal{N}^{*}\left(\mathcal{R}_{i}^{*}\left[\phi_{i}\right]\right)-\prod_{i} \phi_{i}\right\| \\
& \quad \leq \sum_{i=1}^{n}\left\|\mathcal{N}^{*}\left(\mathcal{R}_{i}^{*}\left[\phi_{i}\right]\right)-\phi_{i}\right\| \prod_{j \neq i}\left\|\phi_{j}\right\| \leq n \epsilon \prod_{i=1}^{n}\left\|\phi_{i}\right\| .
\end{aligned}
$$

From Eqs. (B4) and (B5), we see that Eq. (B2) follows immediately by the triangle inequality.

Although we prove this result for general quantum operations on finite-dimensional von Neumann algebras, the special case of ordinary quantum channels between density matrices of finite-dimensional Hilbert spaces follows immediately by considering $\mathcal{A}_{i}=\mathcal{A}=B\left(\mathcal{H}_{A}\right)$ and $\mathcal{B}=B\left(\mathcal{H}_{B}\right)$. Perhaps surprisingly, the result does not appear to have been previously known, even for a single recovery channel and for ordinary subspace quantum-error correction.

To conclude this section, we provide a differential formula for the Choi operator of the recovery map $\mathcal{R}$, which is given in Eq. (8) above,

$$
\Phi_{\mathcal{R}}=\left.\frac{d}{d t}\right|_{t=0} \log \left(\overline{\mathcal{N}[\sigma]} \otimes \sigma^{-1}+t \Phi_{\mathcal{N}^{*}}\right)
$$

where $\mathcal{N}^{*}$ denotes the adjoint of the channel $\mathcal{N}$, and $\overline{\mathcal{N}}[\sigma]$ is the complex conjugate of $\mathcal{N}[\sigma]$. First, recall the integral formula (see, e.g., Ref. [36] Lemma 3.4) 


$$
\left.\frac{d}{d t}\right|_{t=0} \log (A+t B)=\int_{-\infty}^{\infty} d t \beta_{0}(t) A^{-\frac{1}{2}+\frac{i t}{2}} B A^{-\frac{1}{2}-\frac{i t}{2}}
$$

Then we have by direct computation

$$
\begin{aligned}
\left.\frac{d}{d t}\right|_{t=0} & \log \left(\overline{\mathcal{N}[\sigma]} \otimes \sigma^{-1}+t \Phi_{\mathcal{N}^{*}}\right) \\
= & \int_{-\infty}^{\infty} d t \beta_{0}(t)\left(\overline{\mathcal{N}[\sigma]} \otimes \sigma^{-1}\right)^{-\frac{1}{2}+\frac{i t}{2}} \Phi_{\mathcal{N}^{*}}\left(\overline{\mathcal{N}[\sigma]} \otimes \sigma^{-1}\right)^{-\frac{1}{2}-\frac{i t}{2}} \\
& =\int_{-\infty}^{\infty} d t \beta_{0}(t)\left(I \otimes \sigma^{\frac{1}{2}-\frac{i t}{2}}\right)\left((\overline{\mathcal{N}[\sigma]} \otimes I)^{-\frac{1}{2}+\frac{i t}{2}}\left(I \otimes \mathcal{N}^{*}\right)[\Phi](\overline{\mathcal{N}[\sigma]} \otimes I)^{-\frac{1}{2}-\frac{i t}{2}}\right)\left(I \otimes \sigma^{\frac{1}{2}+\frac{i t}{2}}\right) \\
& =\int_{-\infty}^{\infty} d t \beta_{0}(t)\left(I \otimes \sigma^{\frac{1}{2}-\frac{i t}{2}}\right)\left(I \otimes \mathcal{N}^{*}\right)\left[\left(I \otimes \mathcal{N}[\sigma]^{-\frac{1}{2}+\frac{i t}{2}}\right) \Phi\left(I \otimes \mathcal{N}[\sigma]^{-\frac{1}{2}-\frac{i t}{2}}\right)\right]\left(I \otimes \sigma^{\frac{1}{2}+\frac{i t}{2}}\right),
\end{aligned}
$$

which, comparing to Eq. (B1), is indeed $\Phi_{\mathcal{R}}$.

\section{APPENDIX C: ENTANGLEMENT WEDGE RECONSTRUCTION FOR ALGEBRAS}

We are interested in reconstructing bulk operators acting on the entanglement wedge of a subregion of the boundary CFT using only boundary data supported in that subregion. A simplified picture of our setup includes the following data: a boundary CFT modeled by an algebra of observables $\mathcal{M}_{\mathrm{CFT}}$, a subalgebra $\mathcal{M}_{A} \subseteq \mathcal{M}_{\mathrm{CFT}}$ of operators acting on a boundary subregion $A$ of the CFT, a code space modeled by an algebra of bulk observables $\mathcal{M}_{\text {code }}$, and a subalgebra $\mathcal{M}_{a} \subseteq \mathcal{M}_{\text {code }}$ of operators acting inside the entanglement wedge of $A$. We also have a bulk-to-boundary map $\mathcal{J}: S\left(\mathcal{M}_{\text {code }}\right) \rightarrow S\left(\mathcal{M}_{\mathrm{CFT}}\right)$ taking code states in the bulk to states on the boundary. The setup is as follows:
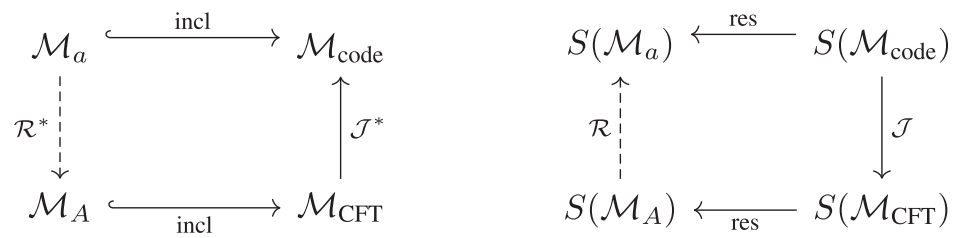

The map $\mathcal{R}^{*}$ (dashed) is the desired map implementing entanglement wedge reconstruction that we construct in Theorem 4 below. A fully general treatment of the problem includes infinite-dimensional algebras of observables. However, there are many technical difficulties in infinite dimensions, and as such, we restrict ourselves to finite-dimensional algebras as in Ref. [31]. Our setup and analysis closely resemble the one used in the main body of this paper, with appropriate changes made to account for the more general algebraic structure.

The following theorem generalizes our main results to the setup above, showing that approximate equality of relative entropies implies approximate entanglement wedge reconstruction even at the level of algebras.

Theorem 4. Let $\mathcal{M}_{a} \subseteq \mathcal{M}_{\text {code }}$ and $\mathcal{M}_{A} \subseteq \mathcal{M}_{\mathrm{CFT}}$ be finite-dimensional von Neumann algebras, $\mathcal{J}: S\left(\mathcal{M}_{\text {code }}\right) \rightarrow$ $S\left(\mathcal{M}_{\mathrm{CFT}}\right)$ a quantum channel, and $\epsilon>0$, such that

$$
\mid D\left(\rho_{a} \| \sigma_{a}\right)-D\left(\mathcal{J}[\rho]_{A} \| \mathcal{J}[\sigma]_{A}\right) \leq \epsilon
$$

for all $\rho, \sigma \in S\left(\mathcal{M}_{\text {code }}\right)$, where we denote by $\rho_{X}$ the restriction $\left.\rho\right|_{\mathcal{M}_{X}}$ of a state $\rho$ to some subalgebra $\mathcal{M}_{X}$. Then, there exists a map $\mathcal{R}: S\left(\mathcal{M}_{A}\right) \rightarrow S\left(\mathcal{M}_{a}\right)$, such that for all $\rho \in S\left(\mathcal{M}_{\text {code }}\right)$ and $\phi_{a}, \phi_{a}^{\prime} \in \mathcal{M}_{a}$,

(i) $\left\|\rho_{a}-\mathcal{R}\left(\mathcal{J}[\rho]_{A}\right)\right\|_{1} \leq \delta$,

(ii) $\left|\left\langle\mathcal{R}^{*}\left[\phi_{a}\right]\right\rangle_{\mathcal{J}[\rho]}-\left\langle\phi_{a}\right\rangle_{\rho}\right| \leq \delta\left\|\phi_{a}\right\|$, 
(iii) $\left|\left\langle\prod_{i=1}^{n} \mathcal{R}^{*}\left[\phi_{i}\right]\right\rangle_{\mathcal{J}[\rho]}-\left\langle\prod_{i=1}^{n} \phi_{i}\right\rangle_{\rho}\right| \leq[\sqrt{2 \ln 2}+(3 n-1) n] \sqrt{\epsilon} \prod_{i}\left\|\phi_{i}\right\|$, where $\delta:=(2+\sqrt{2 \ln 2}) \sqrt{\epsilon}$. Explicitly,

$$
\mathcal{R}^{*}\left[\phi_{a}\right]=\int d t \beta_{0}(t) e^{\frac{1-i t}{2} H_{A}} \mathcal{J}\left[E_{a}\left(e^{-\frac{1-i t}{2} H_{a}} \phi_{a} e^{-\frac{1+i t}{2} H_{a}}\right)\right]_{A} e^{\frac{1+i t}{2} H_{A}},
$$

where $H_{a}=-\log \sigma_{a}$ and $H_{A}=-\log \mathcal{J}\left(\mathcal{E}_{a}\left[\sigma_{a}\right]\right)_{A}$ for some arbitrary fixed full-rank state $\sigma_{a} \in S\left(\mathcal{M}_{a}\right)$, with $\mathcal{E}_{a}$ the state extension map $S\left(\mathcal{M}_{a}\right) \subseteq S\left(\mathcal{M}_{\text {code }}\right)$ from Eq. (A1).

Proof.-We consider the "local" quantum channel

$$
\mathcal{N}: S\left(\mathcal{M}_{a}\right) \rightarrow S\left(\mathcal{M}_{A}\right), \quad \omega_{a} \mapsto \mathcal{J}\left(\mathcal{E}_{a}\left[\omega_{a}\right]\right)_{A} .
$$

A crucial property of $\mathcal{N}$ is that, for any state $\rho \in S\left(\mathcal{M}_{\text {code }}\right)$,

$$
\begin{aligned}
\left\|\mathcal{N}\left[\rho_{a}\right]-\mathcal{J}[\rho]_{A}\right\|_{1} & =\left\|\mathcal{J}\left(\mathcal{E}_{a}\left[\rho_{a}\right]\right)_{A}-\mathcal{J}[\rho]_{A}\right\|_{1} \leq \sqrt{2 \ln 2 D\left(\mathcal{J}\left[\mathcal{E}_{a}\left(\rho_{a}\right)\right]_{A} \| \mathcal{J}[\rho]_{A}\right)} \\
& \leq \sqrt{2 \ln 2\left(D\left[\mathcal{E}_{a}\left(\rho_{a}\right) \|_{a} \rho_{a}\right]+\epsilon\right)}=\sqrt{2 \ln 2 \epsilon}
\end{aligned}
$$

where the first inequality is the familiar relation between trace norm and relative entropy, the second inequality is our assumption Eq. (C1), and the last identity is Eq. (A1).

We now let $\mathcal{R}=\mathcal{R}_{\sigma_{a}, \mathcal{N}}$ denote the recovery map (B1) associated with some full-rank state $\sigma_{a} \in S\left(\mathcal{M}_{a}\right)$. With this choice of $\mathcal{R}$, Eq. (C2) holds true. Moreover, Lemma 2 shows that, for all $\rho \in S\left(\mathcal{M}_{\text {code }}\right)$,

$-2 \log F\left(\rho_{a},(\mathcal{R} \circ \mathcal{N})\left[\rho_{a}\right]\right) \leq D\left(\rho_{a} \| \sigma_{a}\right)-D\left(\mathcal{N}\left[\rho_{a}\right] \| \mathcal{N}\left[\sigma_{a}\right]\right)=D\left(\mathcal{E}_{a}\left[\rho_{a}\right]_{a} \| \mathcal{E}_{a}\left[\sigma_{a}\right]_{a}\right)-D\left(\mathcal{J}\left(\mathcal{E}_{a}\left[\rho_{a}\right]\right)_{A} \| \mathcal{J}\left(\mathcal{E}_{a}\left[\sigma_{a}\right]\right)_{A}\right) \leq \epsilon$

by using our assumption Eqs. (C1) and (A1) a second time. Thus, $F\left(\rho_{a},(\mathcal{R} \circ \mathcal{N})\left[\rho_{a}\right]\right) \geq 1-\epsilon / 2$, and using the Fuchs-van de Graaf inequality,

$$
\left\|\rho_{a}-\mathcal{R}\left(\mathcal{N}\left[\rho_{a}\right]\right)\right\|_{1} \leq 2 \sqrt{1-F\left(\rho_{a},(\mathcal{R} \circ \mathcal{N})\left[\rho_{a}\right]\right)^{2}} \leq 2 \sqrt{\epsilon} .
$$

We obtain (i) from Eqs. (C3) and (C4) and the triangle inequality. This readily implies (ii), since

$$
\left|\left\langle\mathcal{R}^{*}\left[\phi_{a}\right]\right\rangle_{\mathcal{J}[\rho]}-\left\langle\phi_{a}\right\rangle_{\rho}\right|=\left|\left\langle\mathcal{R}^{*}\left[\phi_{a}\right]\right\rangle_{\mathcal{J}[\rho]_{A}}-\left\langle\phi_{a}\right\rangle_{\rho_{a}}\right|=\left|\left\langle\phi_{a}\right\rangle_{\mathcal{R}\left[\mathcal{J}[\rho]_{A}\right]}-\left\langle\phi_{a}\right\rangle_{\rho_{a}}\right| \leq \delta\left\|\phi_{a}\right\| .
$$

For (iii), we observe that

$$
\begin{aligned}
\left|\left\langle\prod_{i} \mathcal{R}^{*}\left[\phi_{i}\right]\right\rangle_{\mathcal{J}[\rho]}-\left\langle\prod_{i} \phi_{i}\right\rangle_{\rho}\right| & =\left|\left\langle\prod_{i} \mathcal{R}^{*}\left[\phi_{i}\right]\right\rangle_{\mathcal{J}[\rho]_{A}}-\left\langle\prod_{i} \phi_{i}\right\rangle_{\rho_{a}}\right| \leq\left|\left\langle\prod_{i} \mathcal{R}^{*}\left[\phi_{i}\right]\right\rangle_{\mathcal{N}\left[\rho_{a}\right]}-\left\langle\prod_{i} \phi_{i}\right\rangle_{\rho_{a}}\right|+\sqrt{2 \ln 2 \epsilon} \prod_{i}\left\|\phi_{i}\right\| \\
& =\left|\left\langle\mathcal{N}^{*}\left(\prod_{i} \mathcal{R}^{*}\left[\phi_{i}\right]\right)\right\rangle_{\rho_{a}}-\left\langle\prod_{i} \phi_{i}\right\rangle_{\rho_{a}}\right|+\sqrt{2 \ln 2 \epsilon} \prod_{i}\left\|\phi_{i}\right\| \\
& \leq[\sqrt{2 \ln 2}+(3 n-1) n] \sqrt{\epsilon} \prod_{i}\left\|\phi_{i}\right\|,
\end{aligned}
$$

where the first inequality is Eq. (C3), and the second inequality is Theorem 3.

When the fiducial state $\sigma_{a} \in S\left(\mathcal{M}_{a}\right)$ is chosen to be the maximally mixed state, then the map (C2) takes a particularly simple form. In this case, $\mathcal{E}_{a}\left[\sigma_{a}\right]=\mathbb{1}_{\text {code }} / d_{\text {code }}=: \tau_{\text {code }}$, where $d_{\text {code }}:=\operatorname{Tr}\left[\mathbb{1}_{\text {code }}\right]$. We obtain 


$$
\mathcal{R}^{*}\left[\phi_{a}\right]=\frac{1}{d_{\text {code }}} \int d t \beta_{0}(t) e^{[(1-i t) / 2] H_{A}} \mathcal{J}\left[\phi_{a}\right]_{A} e^{[(1+i t) / 2] H_{A}},
$$

where we recall that $H_{A}=-\log \mathcal{J}\left[\tau_{\text {code }}\right]_{A}$ is the modular Hamiltonian of the boundary region $A$ associated with the maximally mixed code state. Equation (C5) can be rewritten as follows (Ref. [36] Lemma 3.4):

$$
\begin{aligned}
\mathcal{R}^{*}\left[\phi_{a}\right] & =\left.\frac{1}{d_{\text {code }}} \frac{d}{d t}\right|_{t=0} \log \mathcal{J}\left[\tau_{\text {code }}+t \phi_{a}\right]_{A} \\
& =-\left.\frac{1}{d_{\text {code }}} \frac{d}{d t}\right|_{t=0} H_{A}\left[\tau_{\text {code }}+t \phi_{a}\right],
\end{aligned}
$$

where we introduce the notation $H_{A}[\rho]:=-\log \mathcal{J}[\rho]_{A}$ for the boundary modular Hamiltonian on subregion $A$ associated with a bulk state $\rho \in S\left(\mathcal{M}_{\text {code }}\right)$. That is, the boundary operator $\mathcal{R}^{*}\left[\phi_{a}\right]$ can be found as the response of the boundary modular Hamiltonian to a perturbation of the fiducial bulk state in the direction of the bulk operator $\phi_{a}$.

If $\sigma_{a}$ is not the maximally mixed state but is instead some arbitrary state, Eq. (C6) for $\mathcal{R}^{*}\left[\phi_{a}\right]$ no longer holds. However, using Eq. (B6) we can write a similar equation for the Choi operator of $\mathcal{R}^{*}$ itself:

$$
\Phi_{\mathcal{R}^{*}}=\left.\frac{d}{d t}\right|_{t=0} \log \left[\bar{\sigma}_{a}^{-1} \otimes \mathcal{J}\left(\mathcal{E}_{a}\left(\sigma_{a}\right)\right)_{A}+t \Phi_{\left.\mathcal{J}\left(\mathcal{E}_{a}[]\right]\right)_{A}}\right]
$$

where $\Phi_{\mathcal{J}\left(\mathcal{E}_{a}[\cdot]\right)_{A}}$ is the Choi operator of $\mathcal{J}\left(\mathcal{E}_{a}[\cdot]\right)_{A}$.

Finally, we show that correlation functions of bulk operators are preserved even when each operator is reconstructed using a different entanglement wedge.

Corollary 5. Let $\mathcal{M}_{a_{i}} \subseteq \mathcal{M}_{\text {code }}$ and $\mathcal{M}_{A_{i}} \subseteq \mathcal{M}_{\mathrm{CFT}}$ be sets of finite-dimensional von Neumann algebras, $\mathcal{J}: S\left(\mathcal{M}_{\text {code }}\right) \rightarrow S\left(\mathcal{M}_{\mathrm{CFT}}\right)$ a quantum channel such that, for each pair of algebras $\mathcal{M}_{a_{i}}$ and $\mathcal{M}_{A_{i}}$, the JLMS condition (C1) holds for some $\epsilon>0$. Then,

$$
\begin{aligned}
& \left|\left\langle\prod_{i=1}^{n} \mathcal{R}_{A_{i}}^{*}\left[\phi_{i}\right]\right\rangle_{\mathcal{J}[\rho]}-\left\langle\prod_{i=1}^{n} \phi_{i}\right\rangle_{\rho}\right| \\
& \quad \leq \frac{1}{2} n(3 n-1)(2+\sqrt{2 \ln 2}) \sqrt{\epsilon} \prod_{i=1}^{n}\left\|\phi_{i}\right\|,
\end{aligned}
$$

where the recovery maps $\mathcal{R}_{A_{i}}$ are defined by applying the explicit construction (C2) to the pairs of algebras $\mathcal{M}_{a_{i}}$ and $\mathcal{M}_{A_{i}}$.

Proof.-The proof is a simple consequence of applying Theorem 3 to condition (i) of Theorem 4. If we take $\mathcal{J}$ to be the encoding map (denoted as $\mathcal{N}$ in Theorem 3) and include the restriction onto each boundary subalgebra $\mathcal{M}_{A_{i}}$ as part of the corresponding recovery map $\mathcal{R}_{A_{i}}$, then Eq. (C7) follows immediately.

\section{APPENDIX D: RINDLER WEDGE RECONSTRUCTION FROM GLOBAL RECONSTRUCTION FOR FREE FIELDS}

In this Appendix, we work through an illustrative example by applying our entanglement wedge reconstruction formula in a problem motivated by AdS-Rindler reconstruction using only global HKLL [2] as input. This example is strictly valid only for free-field theories, but we nevertheless use the language of AdS/CFT for familiarity; in short, we pretend both bulk and boundary fields are free, and we comment on the difficulties that arise when the boundary field is only a generalized free field. Let $A$ be the boundary region corresponding to a single Rindler wedge, $a$ be the entanglement wedge of $A, D_{A}$ be the boundary domain of dependence of $A$, and $\bar{A}$ be the complement of $A$. We show that local bulk operators in $a$ can be represented as linear combinations of field operators for Rindler modes confined to region $A$. The subtlety for a true AdS/CFT calculation lies in the Rindler decomposition; in general, no such decomposition exists for generalized free fields. 
For simplicity, we consider the case of $\mathrm{AdS}_{3}$. We use Poincaré patch coordinates,

$$
d s^{2}=\frac{\ell^{2}}{z^{2}}\left(-d t^{2}+d x^{2}+d z^{2}\right)
$$

and we label bulk points by $Y=(t, x, z)$ and boundary points by $y=(t, x)$.

Suppose that we want to reconstruct a bulk operator $\phi(Y)$ for $Y \in a$ on the boundary of the Rindler wedge $A$. Let us denote the vacuum state by $|\tilde{0}\rangle$ and an excitation of the ground state by $|\tilde{1}\rangle=\phi(Y)|\tilde{0}\rangle$, which we take to be normalized. We consider a two-dimensional code space $\mathcal{H}_{\text {code }}=\operatorname{span}\{|\tilde{0}\rangle,|\tilde{1}\rangle\}$. Our goal is to reconstruct the action of the operator $\phi(Y)$ on the code space restricted to the boundary interval $A$. The maximally mixed state on the code is simply $\tau=\frac{1}{2}(|\tilde{0}\rangle\langle\tilde{0}|+| \tilde{1}\rangle\langle\tilde{1}|)$. Note that in this simple example $\mathcal{H}_{a}=\mathcal{H}_{\text {code }}$, since we do not consider any d.o.f. living in $\bar{a}$.

As an operator on the code space, $\phi(Y)$ acts as $X:=|\tilde{1}\rangle\langle\tilde{0}|+$ H.c., mapping the vacuum state to the excited state and vice versa. With our chosen operator, code space, and maximally mixed state, we can rewrite Eq. (16) as

$$
\begin{aligned}
\mathcal{R}^{*}[X]= & \frac{1}{2} \int d t \beta_{0}(t) \mathcal{N}[\tau]^{[(-1+i t) / 2]} \mathcal{N}[|\tilde{1}\rangle\langle\tilde{0}|+| \tilde{0}\rangle\langle\tilde{1}|] \\
& \times \mathcal{N}[\tau]^{-[(1+i t) / 2]},
\end{aligned}
$$

where we introduce $\mathcal{N}\left[\rho_{a}\right]=\operatorname{Tr}_{\bar{A}}\left[J \rho_{a} J^{\dagger}\right]$ as shorthand.

In order to evaluate Eq. (D1), we need to compute terms of the form

$$
\mathcal{N}(|\tilde{x}\rangle\langle\tilde{y}|)=\operatorname{Tr}_{\bar{A}}[|x\rangle\langle y|]
$$

where $x, y \in\{0,1\}$, and the states $|x\rangle:=J|\tilde{x}\rangle$. The empty AdS state $|\tilde{0}\rangle$ is mapped to the CFT ground state $J|\tilde{0}\rangle=|0\rangle$. The excited state $|\tilde{1}\rangle$ is mapped via global HKLL to

$$
J|\tilde{1}\rangle=:|1\rangle=\int_{y^{\prime} \in D} d y^{\prime} K_{g}\left(Y, y^{\prime}\right) \Phi\left(y^{\prime}\right)|0\rangle,
$$

where $K_{g}$ is a bulk-to-boundary kernel ( $g$ denotes "global"), $\Phi(y)$ is a boundary operator, and $D$ is a boundary spacetime domain.

To leading order in $1 / N, \Phi(y)$ behaves like a generalized free field [37]. Unfortunately, generalized free fields do not, in general, admit a decomposition into Rindler modes. Thus, we now pretend the boundary field is instead a true free field, and we expand it in terms of Rindler modes $\left\{a_{\ell}, b_{\ell}\right\}$ adapted to $A$ and $B=\bar{A}$ :

$$
\Phi(y)=\sum_{\ell} f_{a, \ell}(y) a_{\ell}+f_{a, \ell}^{*}(y) a_{\ell}^{\dagger}+f_{b, \ell}(y) b_{\ell}+f_{b, \ell}^{*}(y) b_{\ell}^{\dagger} .
$$

In order to upgrade this calculation to a true AdS/CFT computation, some care needs to be taken with respect to this decomposition for generalized free fields, but we nevertheless forge ahead in the name of pedagogy. Note that the modes $a_{\ell}$ and $b_{\ell}$ are entangled in the state $|0\rangle$ since there is entanglement between $A$ and $B$.

With the HKLL prescription of $|0\rangle=J|\tilde{0}\rangle$ and $|1\rangle=J|\tilde{1}\rangle$ in hand, we can now compute the partial trace of the various matrix elements appearing in Eq. (D1) with respect to the region $\bar{A}$. We begin with $\operatorname{Tr}_{B}[|0\rangle\langle 0|+| 1\rangle\langle 1|]$. Since $|0\rangle$ and $|1\rangle$ are approximately distinguishable on $A$, the result of tracing out $\bar{A}$ (i.e., tracing out the $B$ modes) will be approximately block diagonal. On the upper block of the reduced density matrix, we have the ground-state density matrix $\rho_{A, 0}=\operatorname{Tr}_{B}[|0\rangle\langle 0|]$ for $A$, and on the lower block, we have

$$
\rho_{A, 1}=\operatorname{Tr}_{\bar{A}}[|1\rangle\langle 1|]=\operatorname{Tr}_{\bar{A}}\left[\int_{y_{1} \in D} \int_{y_{2} \in D} K_{g}\left(Y, y_{1}\right) K_{g}\left(Y, y_{2}\right) \Phi\left(y_{1}\right)|0\rangle\langle 0| \Phi\left(y_{2}\right)\right] .
$$


To simplify the form of $\rho_{A, 1}$, a crucial fact is that the ground state $|0\rangle$ is comprised of entangled $a_{\ell}$ and $b_{\ell}$ modes. More precisely, focusing on a single mode, there is a modular energy $E_{\ell}$ such that the ground state is of the form

$$
|0\rangle \propto \sum_{n} e^{-\pi E_{\ell} n}|n\rangle_{a_{\ell}}|n\rangle_{b_{\ell}}
$$

In such a state, the reduced density matrix of $A$ has the form $\rho_{A, 0} \propto \sum_{n} e^{-2 \pi E_{\ell} n}|n\rangle\left\langle\left. n\right|_{a_{\ell}}\right.$, so that

$$
|0\rangle \propto \rho_{A, 0}^{1 / 2} \sum_{n}|n\rangle_{a_{\ell}}|n\rangle_{b_{\ell}}
$$

Using the "transpose trick," it follows that mode operators on $\bar{A}$ can be written in terms of operators on $A$ :

$$
\begin{aligned}
& b_{\ell}|0\rangle=\rho_{A, 0}^{1 / 2} a_{\ell}^{\dagger} \rho_{A, 0}^{-1 / 2}|0\rangle, \\
& b_{\ell}^{\dagger}|0\rangle=\rho_{A, 0}^{1 / 2} a_{\ell} \rho_{A, 0}^{-1 / 2}|0\rangle,
\end{aligned}
$$

and vice versa. We also note the following helpful identities:

$$
\begin{aligned}
& \rho_{A, 0}^{1 / 2} a_{\ell} \rho_{A, 0}^{-1 / 2}=a_{\ell} e^{\pi E_{\ell}}, \\
& \rho_{A, 0}^{-1 / 2} a_{\ell} \rho_{A, 0}^{1 / 2}=a_{\ell} e^{-\pi E_{\ell}}, \\
& \rho_{A, 0}^{1 / 2} a_{\ell}^{\dagger} \rho_{A, 0}^{-1 / 2}=a_{\ell}^{\dagger} e^{-\pi E_{\ell}}, \\
& \rho_{A, 0}^{-1 / 2} a_{\ell}^{\dagger} \rho_{A, 0}^{1 / 2}=a_{\ell}^{\dagger} e^{\pi E_{\ell}} .
\end{aligned}
$$

Using Eq. (D2), we now evaluate $\mathcal{N}[|\tilde{1}\rangle\langle\tilde{0}|]=\operatorname{Tr}_{\bar{A}}[|1\rangle\langle 0|]$ :

$$
\mathcal{N}[|\tilde{1}\rangle\langle\tilde{0}|]=\int_{y \in D} K_{g}(Y, y) \operatorname{Tr}_{\bar{A}}\left[\left(\sum_{\ell} f_{a, \ell}(y) a_{\ell}+f_{a, \ell}^{*}(y) a_{\ell}^{\dagger}+f_{b, \ell}(y) b_{\ell}+f_{b, \ell}^{*}(y) b_{\ell}^{\dagger}\right)|0\rangle\langle 0|\right] .
$$

For brevity, let

$$
\begin{aligned}
& \check{f}_{a, \ell}=\int_{y \in D} K_{g}(Y, y) f_{\ell, a}(y), \\
& \check{f}_{b, \ell}=\int_{y \in D} K_{g}(Y, y) f_{\ell, b}(y) .
\end{aligned}
$$

Using Eq. (D3), we can rewrite $\mathcal{N}[|\tilde{1}\rangle\langle\tilde{0}|]=\int K_{g} \Phi|0\rangle\left\langle 0\left|=Q_{A}\right| 0\right\rangle\langle 0|$, where

$$
Q_{A}=\sum_{\ell} \check{f}_{a, \ell} a_{\ell}+\check{f}_{a, \ell}^{*} a_{\ell}^{\dagger}+\check{f}_{b, \ell} \rho_{A, 0}^{1 / 2} a_{\ell}^{\dagger} \rho_{A, 0}^{-1 / 2}+\check{f}_{b, \ell}^{*} \rho_{A, 0}^{1 / 2} a_{\ell} \rho_{A, 0}^{-1 / 2} .
$$

Finally, using the identities (D4), we write $Q_{A}$ as

$$
Q_{A}=\sum_{\ell} \check{f}_{a, \ell} a_{\ell}+\check{f}_{a, \ell}^{*} a_{\ell}^{\dagger}+\check{f}_{b, \ell} a_{\ell}^{\dagger} e^{-\pi E_{\ell}}+\check{f}_{b, \ell}^{*} a_{\ell} e^{\pi E_{\ell}},
$$

taking note that this operator is only guaranteed to reproduce the action of $\int K_{g} \Phi$ when acting on $|0\rangle$. 
Using $Q_{A}$, we can write $\mathcal{N}[|\tilde{x}\rangle\langle\tilde{y}|]=\left(Q_{A}\right)^{x} \rho_{A, 0}\left(Q_{A}^{\dagger}\right)^{y}$, where $x, y \in\{0,1\}$. In particular, we have that $\rho_{A, 1}=Q_{A} \rho_{A, 0} Q_{A}^{\dagger}$ and $\mathcal{N}[|\tilde{1}\rangle\langle\tilde{0}|]=Q_{A} \rho_{A, 0}$. We then rewrite $Q_{A} \rho_{A, 0}=\rho_{A, 0}^{1 / 2}\left(\rho_{A, 0}^{-1 / 2} Q_{A} \rho_{A, 0}^{1 / 2}\right) \rho_{A, 0}^{1 / 2}$, where the term in the parentheses is still a simple sum of $a_{\ell}$ and $a_{\ell}^{\dagger}$ with various weights. Explicitly, it is

$$
\begin{aligned}
\rho_{A, 0}^{-1 / 2} Q_{A} \rho_{A, 0}^{1 / 2}= & \sum_{\ell} \check{f}_{a, \ell} a_{\ell} e^{-\pi E_{\ell}}+\check{f}_{a, \ell}^{*} a_{\ell}^{\dagger} e^{\pi E_{\ell}} \\
& +\check{f}_{b, \ell} a_{\ell}^{\dagger}+\check{f}_{b, \ell}^{*} a_{\ell} .
\end{aligned}
$$

Using the approximate block diagonality of $\mathcal{N}[\tau]$, we can write

$$
\mathcal{N}[\tau]^{[(-1+i t) / 2]} \approx\left(\frac{1}{2}\right)^{[(1-i t) / 2]}\left(\rho_{A, 0}^{\frac{-1+i t}{2}}+\rho_{A, 1}^{\frac{-1+i t}{2}}\right),
$$

although the reader is cautioned that we have not analyzed the quality of this approximation. The approximate block diagonality also implies that

$$
\begin{aligned}
\mathcal{N}[\tau]^{[(-1+i t) / 2]} \rho_{A, 0}^{1 / 2} & \approx\left(\frac{1}{2}\right)^{[(1-i t) / 2]} \rho_{A, 0}^{\frac{-1+i t}{2}} \rho_{A, 0}^{1 / 2} \\
& =\left(\frac{1}{2}\right)^{[(1-i t) / 2]} \rho_{A, 0}^{i t / 2},
\end{aligned}
$$

and a similar argument shows that

$$
\rho_{A, 0}^{1 / 2} \mathcal{N}[\tau]^{[(-1-i t) / 2]} \approx\left(\frac{1}{2}\right)^{[(1+i t) / 2]} \rho_{A, 0}^{-i t / 2}
$$

Thus, the recovery channel is proportional to

$$
\mathcal{R}^{*}[|\tilde{1}\rangle\langle\tilde{0}|]=\int d t \beta_{0}(t) \rho_{A, 0}^{i t / 2}\left(\rho_{A, 0}^{-1 / 2} Q_{A} \rho_{A, 0}^{1 / 2}\right) \rho_{A, 0}^{i t / 2},
$$

and we note that the factors of $1 / 2$ cancel out. The combined object $\rho_{A, 0}^{i t / 2}\left(\rho_{A, 0}^{-1 / 2} Q_{A} \rho_{A, 0}^{1 / 2}\right) \rho_{A, 0}^{-i t / 2}$ is then

$$
\begin{gathered}
\sum_{\ell} \check{f}_{a, \ell} a_{\ell} e^{-\pi E_{\ell}+i \pi E_{\ell} t}+\check{f}_{a, \ell}^{*} a_{\ell}^{\dagger} e^{\pi E_{\ell}-i \pi E_{\ell} t} \\
+\check{f}_{b, \ell} a_{\ell}^{\dagger} e^{-i \pi E_{\ell} t}+\check{f}_{b, \ell}^{*} a_{\ell} e^{i \pi E_{\ell} t}
\end{gathered}
$$

Defining $\hat{\beta}_{0}(\omega):=\int d t \beta_{0}(t) e^{i \omega t}$ and noting that $\hat{\beta}_{0}(-\omega)=\hat{\beta}_{0}(\omega)$ by symmetry of $\beta_{0}(t)$, the recovery map acting on our operator is

$$
\begin{aligned}
\mathcal{R}^{*}[|\tilde{1}\rangle\langle\tilde{0}|]= & \sum_{\ell} \check{f}_{a, \ell} a_{\ell} e^{-\pi E_{\ell}} \hat{\beta}_{0}\left(\pi E_{\ell}\right) \\
& +\check{f}_{a, \ell}^{*} a_{\ell}^{\dagger} e^{\pi E_{\ell}} \hat{\beta}_{0}\left(\pi E_{\ell}\right)+\check{f}_{b, \ell} a_{\ell}^{\dagger} \hat{\beta}_{0}\left(\pi E_{\ell}\right) \\
& +\check{f}_{b, \ell}^{*} a_{\ell} \hat{\beta}_{0}\left(\pi E_{\ell}\right),
\end{aligned}
$$

and there is an analogous expression for $\mathcal{R}^{*}[|\tilde{0}\rangle\langle\tilde{1}|]$.

Equation (D5) is our desired result. The bulk operator $X=|\tilde{1}\rangle\langle\tilde{0}|+$ H.c. can be reconstructed on the Rindler wedge using only Rindler mode operators. Moreover, only single-mode operators appear. 
[1] J. M. Maldacena, The Large $N$ Limit of Superconformal Field Theories and Supergravity, Int. J. Theor. Phys. 38, 1113 (1999); Adv. Theor. Math. Phys. 2, 231 (1998).

[2] A. Hamilton, D. Kabat, G. Lifschytz, and D. A. Lowe, Holographic Representation of Local Bulk Operators, Phys. Rev. D 74, 066009 (2006).

[3] A. Hamilton, D. Kabat, G. Lifschytz, and D. A. Lowe, Local Bulk Operators in AdS/CFT Correspondence: A Boundary View of Horizons and Locality, Phys. Rev. D 73, 086003 (2006).

[4] A. Hamilton, D. Kabat, G. Lifschytz, and D. A. Lowe, Local Bulk Operators in AdS/CFT Correspondence: A Holographic Description of the Black Hole Interior, Phys. Rev. D 75, 106001 (2007).

[5] D. Kabat, G. Lifschytz, and D. A. Lowe, Constructing Local Bulk Observables in Interacting AdS/CFT, Phys. Rev. D 83, 106009 (2011).

[6] B. Czech, J. L. Karczmarek, F. Nogueira, and M. Van Raamsdonk, The Gravity Dual of a Density Matrix, Classical Quantum Gravity 29, 155009 (2012).

[7] B. Czech, J. L. Karczmarek, F. Nogueira, and M. Van Raamsdonk, Rindler Quantum Gravity, Classical Quantum Gravity 29, 235025 (2012).

[8] S. Ryu and T. Takayanagi, Holographic Derivation of Entanglement Entropy from the Anti-de Sitter Spacel Conformal Field Theory Correspondence, Phys. Rev. Lett. 96, 181602 (2006).

[9] V.E. Hubeny, M. Rangamani, and T. Takayanagi, A Covariant Holographic Entanglement Entropy Proposal, J. High Energy Phys. 07 (2007) 062.

[10] M. Headrick, V.E. Hubeny, A. Lawrence, and M. Rangamani, Causality \& Holographic Entanglement Entropy, J. High Energy Phys. 12 (2014) 162.

[11] A. C. Wall, Maximin Surfaces, and the Strong Subadditivity of the Covariant Holographic Entanglement Entropy, Classical Quantum Gravity 31, 225007 (2014).

[12] F. Pastawski, B. Yoshida, D. Harlow, and J. Preskill, Holographic Quantum Error-Correcting Codes: Toy Models for the Bulk/Boundary Correspondence, J. High Energy Phys. 06 (2015) 149.

[13] P. Hayden, S. Nezami, X.-L. Qi, N. Thomas, M. Walter, and Z. Yang, Holographic Duality from Random Tensor Networks, J. High Energy Phys. 11 (2016) 009.

[14] S. Nezami and M. Walter, Multipartite Entanglement in Stabilizer Tensor Networks, arXiv:1608.02595.

[15] X. Dong, D. Harlow, and A. C. Wall, Reconstruction of Bulk Operators within the Entanglement Wedge in GaugeGravity Duality, Phys. Rev. Lett. 117, 021601 (2016).

[16] D. L. Jafferis, A. Lewkowycz, J. Maldacena, and S. J. Suh, Relative Entropy Equals Bulk Relative Entropy, J. High Energy Phys. 06 (2016) 004.

[17] P. Hayden, R. Jozsa, D. Petz, and A. Winter, Structure of States Which Satisfy Strong Subadditivity of Quantum Entropy with Equality, Commun. Math. Phys. 246, 359 (2004).
[18] B. Ibinson, N. Linden, and A. Winter, Robustness of Quantum Markov Chains, Commun. Math. Phys. 277, 289 (2008).

[19] D. Ding, P. Hayden, and M. Walter, Conditional Mutual Information of Bipartite Unitaries and Scrambling, J. High Energy Phys. 12 (2016) 145.

[20] P. Hayden and G. Penington, Approximate Quantum Error Correction Revisited: Introducing the Alpha-Bit, arXiv:1706.09434.

[21] P. Hayden and A. Winter, Weak Decoupling Duality and Quantum Identification, IEEE Trans. Inf. Theory 58, 4914 (2012).

[22] P. Hayden and G. Penington, Learning the Alpha-Bits of Black Holes, arXiv:1807.06041.

[23] D. Petz, Sufficient Subalgebras and the Relative Entropy of States of a von Neumann Algebra, Commun. Math. Phys. 105, 123 (1986).

[24] A. Winter and K. Li, A Stronger Subadditivity Relation? With Applications to Squashed Entanglement, Sharability and Separability, in Proceedings of the BIRS Workshop, 2012.

[25] M. Junge, R. Renner, D. Sutter, M. M. Wilde, and A. Winter, Universal Recovery from a Decrease of Quantum Relative Entropy, Ann. Inst. Henri Poincaré 19, 2955 (2018).

[26] M. Ohya and D. Petz, Quantum Entropy and Its Use (Springer, New York, 2004).

[27] S. S. Gubser, I. R. Klebanov, and A. M. Polyakov, Gauge Theory Correlators from Non-Critical String Theory, Phys. Lett. B 428, 105 (1998).

[28] E. Witten, Anti de Sitter Space and Holography, Adv. Theor. Math. Phys. 2, 253 (1998).

[29] T. Banks, M. R. Douglas, G. T. Horowitz, and E. Martinec, AdS Dynamics from Conformal Field Theory, arXiv:hep-th/ 9808016.

[30] A. Almheiri, X. Dong, and D. Harlow, Bulk Locality and Quantum Error Correction in AdS/CFT, J. High Energy Phys. 04 (2015) 163.

[31] D. Harlow, The Ryu-Takayanagi Formula from Quantum Error Correction, arXiv:1607.03901.

[32] C. A. Fuchs and J. van de Graaf, Cryptographic Distinguishability Measures for Quantum-Mechanical States, IEEE Trans. Inf. Theory 45, 1216 (1999).

[33] T. Faulkner and A. Lewkowycz, Bulk Locality from Modular Flow, J. High Energy Phys. 17 (2017) 151.

[34] C.-F. Chen, G. Penington, and G. Salton, Entanglement Wedge Reconstruction using the Petz Map, arXiv: 1902.02844

[35] D. Petz, Quantum Information Theory and Quantum Statistics (Springer, New York, 2007).

[36] D. Sutter, M. Berta, and M. Tomamichel, Multivariate Trace Inequalities, Commun. Math. Phys. 352, 37 (2017).

[37] O. Greenberg, Generalized Free Fields and Models of Local Field Theory, Ann. Phys. (N.Y.) 16, 158 (1961). 